\title{
LOS RECIENTES CAMBIOS EN LA DESIGUALDAD Y LA POBREZA EN CHILE*
}

\section{Osvaldo Larrañaga y Rodrigo Herrera}

El trabajo tiene por objetivo analizar los cambios en la distribución de ingresos y en la pobreza a la luz de los resultados de la encuesta Casen 2006. Los datos muestran que se ha producido un descenso en la desigualdad de ingresos en el período 2000 a 2006, el cual se refleja en todos los indicadores de desigualdad y a nivel de los subperíodos 2000 a 2003 y 2003 a 2006. Las principales causas subyacentes son la reducción de la desigualdad salarial entre trabajadores con distinto nivel de escolaridad, una menor dispersión de los salarios al interior de las categorías educacionales, y un aumento en el ingreso per cápita de los hogares de los deciles inferiores por causa del impacto que la baja en la tasa de natalidad está teniendo en el tamaño de los hogares. La reducción en la desigualdad ha contribuido a la caída de la pobreza, contrastando con la experiencia de los años 90 cuando la caída en la pobreza estaba asociada a un efecto crecimiento que era parejo a lo largo de la distribución de ingresos.

Osvaldo Larrañaga. Ph. D. en Economía, Universidad de Pennsylvania. Ingeniero Comercial, Universidad de Chile. Profesor Asociado, Departamento de Economía de la Universidad de Chile y asesor de política social en el PNUD.

Rodrigo Herrera. Magister en Economía, Universidad de Chile. Ingeniero Comercial, Universidad Tecnológica Metropolitana. Profesional de la División Social de Mideplan.

* Los autores agradecen los comentarios recibidos en seminarios efectuados en Mideplan y en el PNUD, así como los recibidos en una presentación preliminar en la reunión de la Sociedad de Economía de Chile del año 2007. También se agradecen los comentarios de un árbitro de Estudios Públicos. No obstante lo anterior, los contenidos del artículo son de exclusiva responsabilidad de los autores.

Estudios Públicos, 109 (verano 2008). 


\section{Introducción}

\section{I}

La desigualdad de ingreseses es uno de los problemas más importantes de la sociedad chilena. A inicios del 2000 el país presentaba una brecha de 38 veces entre el ingreso medio del 10\% más rico y $10 \%$ más pobre de la población, situándose entre los países más desiguales de una región que se caracteriza por tener malos indicadores en la materia (De Ferrantis et al., 2003) ${ }^{1}$. La citada brecha de ingresos refleja la existencia de enormes diferencias de bienestar entre los hogares del país, reflejo de desigualdades en planos tales como las oportunidades, dotación de capital humano, acceso a activos productivos, etc.

La desigualdad socioeconómica importa por dos tipos de consideraciones. En primer término, una sociedad que sea percibida como injusta es inherentemente inestable, puesto que no provee reglas de cooperación social que sean aceptadas por sus miembros. La desigualdad es injusta si existen diferencias sustanciales en la calidad de vida de las personas que se originan en circunstancias arbitrarias, que no están bajo el control de los individuos, pero que podrían ser modificadas a través de la acción pública (Rawls, 1971). Éstas incluyen la desigual distribución de las oportunidades, la discriminación en el acceso a puestos de estudio y de trabajo, la exclusión social, entre otras materias.

En segundo lugar, en una sociedad desigual no se aprovecha el potencial productivo de parte de sus recursos humanos. Por tanto, la desigualdad supone un uso ineficiente de los recursos productivos en una sociedad y un consiguiente deterioro en las expectativas de desarrollo económico. De hecho, no hay ningún país desarrollado que exhiba, ni cercanamente, los niveles de desigualdad que caracterizan a los países de América Latina.

La persistencia de la desigualdad de ingresos en el país contrasta con los avances que se registran en términos de crecimiento económico y reducción de la pobreza, convirtiéndola en uno de los principales cuestionamientos que se realizan al modelo de desarrollo en vigencia. La situación es particularmente frustrante si se considera que desde 1990 los gobiernos de la Concertación han puesto la equidad como un objetivo central de la estrategia de desarrollo, y que los recursos destinados al gasto social prácticamente se triplican entre 1990 y $2005^{2}$.

\footnotetext{
${ }^{1}$ Los ingresos referidos corresponden al ingreso per cápita de los hogares.

${ }^{2}$ En Larrañaga (2007a) se argumenta que la política social ha carecido de instrumentos que tengan como objetivo explícito la reducción de la desigualdad de ingresos.
} 
Es en tal contexto que la encuesta Casen del año 2006 muestra una fuerte y sorpresiva caída de la desigualdad de los ingresos. La brecha de ingresos entre los deciles más rico y más pobre cae de 38,0 veces en 2000 a 28,5 en el 2006; mientras que el coeficiente de Gini se reduce a 52,2 puntos, después de haber fluctuado en el entorno de 55 puntos en la década del $90^{3}$.

El objetivo de este artículo es documentar la reciente caída en la desigualdad y explorar sus causas subyacentes, de modo de establecer si hay fundamentos sólidos detrás de tal desarrollo. Tal propósito tiene particular relevancia dado que ha habido cuestionamientos de los resultados de la encuesta Casen 2006, relacionados con algunos cambios que se efectuaron en el procedimiento muestral.

El artículo muestra que la reducción de la desigualdad es un hecho robusto, con dos principales causas subyacentes. En primer término, hay una caída significativa en la desigualdad salarial, tanto porque se reduce la brecha de salarios entre trabajadores con distinto nivel de escolaridad como porque se comprime la desigualdad salarial al interior de grupos de ocupados con igual nivel de escolaridad. Los salarios representan más de la mitad del ingreso de los hogares, de manera que cambios en la distribución de salarios tienen un impacto significativo en la distribución del ingreso.

En segundo lugar, hay una caída en el tamaño promedio de los hogares en la mitad inferior de la distribución, lo cual explica por sí sola alrededor de la tercera parte del incremento del ingreso per cápita de estos hogares en el período reciente. Tal desarrollo está relacionado con la fuerte baja en la tasa de fecundidad en las últimas décadas, la cual ha sido particularmente importante en el caso de las mujeres de estrato socioeconómico bajo.

No hay razones aparentes para que estos resultados hayan sido influenciados por los cambios muestrales efectuados en la encuesta Casen 2006. Más aún, la caída de la desigualdad no está acotada a los datos de ese año, sino que ya estaba presente en la medición realizada el año 2003.

Otro resultado importante es que la reducción de la desigualdad contribuye decisivamente a la caída de la pobreza en el período reciente. A diferencia de lo ocurrido en la década de los 90, cuando hay un crecimiento parejo del ingreso de los hogares en los distintos deciles, en el período reciente la caída de la pobreza se origina en el mayor crecimiento del ingreso de los hogares en la mitad inferior de la distribución.

El artículo se organiza en cuatro secciones principales, además de esta introducción. El contenido respectivo de las secciones es: la documen-

\footnotetext{
${ }^{3}$ El cálculo de las estadísticas se describe en la sección 2. Puede haber diferencias menores respecto de otras estimaciones, dependiendo de las variantes metodológicas utilizadas.
} 
tación de la caída de la desigualdad; los cambios en la pobreza y su relación con la desigualdad; el rol de los salarios en la caída de la desigualdad y el papel que desempeñan los desarrollos demográficos. Una última sección presenta las conclusiones.

\section{La desigualdad de ingresos}

Hay dos características de las mediciones de la desigualdad de ingresos que tienen particular relevancia en la discusión que se realiza en este

artículo. Éstas son la naturaleza transversal de las encuestas de hogares y el hecho que los indicadores de desigualdad son anónimos.

La desigualdad de ingresos se mide en base a datos provenientes de encuestas socioeconómicas de hogares, las cuales entrevistan a muestras representativas de la población que son específicas a cada período de medición. De tal manera, las encuestas proveen "fotografías" que describen la realidad socioeconómica en un determinado momento del tiempo. La comparación de encuestas a través del tiempo posibilita conocer la evolución de las variables socioeconómicas, puesto que cada muestra es representativa del conjunto de la población.

Por su parte, los indicadores que miden la desigualdad sólo utilizan la información de ingresos, sin importar su historia o si han sido adquiridos a través de medios justos. Por tal razón, los indicadores de desigualdad son "anónimos".

A modo de ejemplo considérese la situación hipotética en la Tabla $\mathrm{N}^{\circ} 1$. Éste es un país donde hay cinco hogares, cuyo nivel de ingresos se evalúa en dos períodos de tiempo. Cada hogar corresponde a un quintil (20\% del total), ordenados desde el más pobre al más rico. La relación de ingresos entre el quintil más rico y el quintil más pobre es 10 veces (100 vs 10 ) en el primer período y 5 veces ( 50 vs 10 ) en el segundo período. En este país la desigualdad de ingresos ha caído en el tiempo.

La medición descrita de la desigualdad está basada sólo en la información de ingresos. La medida no considera que hay hogares que han cambiado su lugar en la distribución, como es el caso de A y D, que avanzan en la escala relativa de ingresos. Tampoco informan si el proceso que genera la distribución de ingresos es justo o injusto. Más importante para los fines del presente artículo, que el ingreso del primer quintil se mantenga constante en el tiempo no significa que los hogares que constituían el primer quintil hayan mantenido constante su nivel de ingreso. De hecho, el hogar A duplica su ingreso entre ambos períodos. Una similar interpretación puede realizarse para los restantes quintiles. 
TABLA N ${ }^{\circ}$ 1: $\quad$ LA MEDICIÓN DE LA DESIGUALDAD

\begin{tabular}{|c|c|c|c|}
\hline \multicolumn{2}{|c|}{ Año 1} & \multicolumn{2}{|c|}{ Año 2} \\
\hline Hogar & Ingreso & Hogar & Ingreso \\
\hline A & 10 & $\mathrm{C}$ & 10 \\
\hline B & 30 & A & 20 \\
\hline $\mathrm{C}$ & 50 & B & 30 \\
\hline $\mathrm{D}$ & 70 & $\mathrm{E}$ & 40 \\
\hline $\mathrm{E}$ & 100 & $\mathrm{D}$ & 50 \\
\hline
\end{tabular}

De tal forma, la medición de la desigualdad consiste solamente en evaluar las distancias en el ranking de los ingresos. Esta propiedad de los indicadores de desigualdad no está asociada a las encuestas de tipo transversal; puesto que la medición sería la misma si se utilizaran encuestas de tipo panel o longitudinal. Un asunto distinto es que éstas provean información adicional, como es la movilidad del ingreso en el tiempo.

Los datos de desigualdad no tienen un contenido normativo, puesto que no informan si la desigualdad existente es justa o injusta. La literatura sobre teorías de justicia generalmente califica como injustas las situaciones que se originan en factores no controlables por los individuos y que les son perjudiciales (Haussman y MacPherson, 1996). Ahora bien, es más que probable que una desigualdad elevada esté asociada a situaciones de injusticia, pero tal situación no es informada por los indicadores de desigualdad propiamente tales.

La desigualdad de ingresos en el período 1990-2006

En el Cuadro $\mathrm{N}^{0} 1$ se presentan los principales indicadores de la distribución de ingresos en Chile en el período 1990-2006. Estos consisten en parámetros que sintetizan la información del conjunto de los ingresos, como es el caso del coeficiente de Gini ${ }^{4}$. Alternativamente, se utilizan indicadores que miden la distancia de ingresos entre distintos puntos específicos de la distribución, como es la razón de quintiles 5 a 1 (Q5/Q1), la razón de deciles 10 a 1 (D10/D1) y entre los percentil 90 y 10 (P90/P10).

\footnotetext{
${ }^{4}$ El coeficiente de Gini es un promedio ponderado del ingreso de los hogares, donde cada hogar tiene una ponderación que es inversa a su orden en el ranking de ingresos (el hogar de menor ingreso tiene el mayor peso en el promedio; aquel con mayor ingresos tiene el peso más bajo). Véase Sen (1997).
} 
CUADRO N ${ }^{\circ}$ 1: $\quad$ INDICADORES DE DESIGUALDAD: INGRESO PER CÁPITA HOGARES 19902006

\begin{tabular}{lrrrr}
\hline Año & Q5/Q1 & D10/D1 & P90/P10 & Gini \\
\hline 1990 & 16,9 & 36,0 & 10,6 & 55,2 \\
1996 & 17,1 & 35,3 & 11,0 & 55,1 \\
2000 & 17,5 & 38,0 & 10,6 & 55,8 \\
2003 & 16,2 & 34,6 & 9,8 & 54,9 \\
2006 & 14,1 & 28,5 & 9,1 & 52,2 \\
\hline
\end{tabular}

Fuente: Cálculos basados en encuestas Casen, años respectivos.

Toda la información de ingresos que se presenta en el artículo proviene de las encuestas Casen de los años respectivos. Ésta es una encuesta de hogares multi-tópico de gran tamaño muestral (75.000 hogares en el año 2006) y cuyos resultados dan lugar a la mayor parte de las estadísticas sobre distribución de ingresos, pobreza e incidencia del gasto social en el país. Cabe señalar que la versión del año 2006 de la encuesta estuvo sujeta a cambios muestrales que consistieron en una reducción del número de hogares que se entrevistan en una unidad primaria de la muestra (secciones), así como una selección aleatoria de las viviendas que son encuestadas al interior de cada segmento. El propósito de esta modificación es aumentar la precisión de los estimadores a partir de aumentar la representatividad de la muestra en relación a la alternativa de encuestar grupos de viviendas contiguas en cada segmento. El citado cambio muestral debiera reducir la varianza de los estimadores, sin afectar el valor esperado de los mismos ${ }^{5}$.

La variable de análisis es el ingreso per cápita del hogar, que consiste en la suma de los ingresos monetarios y la renta imputada por la vivienda propia, dividido por el total de personas que viven en el hogar. El servicio doméstico que reside en el lugar de trabajo se considera un hogar aparte. Los indicadores se calculan sobre la base de individuos, de modo que un hogar es representado en las estadísticas por cada uno de sus integrantes ${ }^{6}$.

\footnotetext{
${ }^{5}$ Una presentación detallada del cambio muestral se puede encontrar en Mideplan (2007).

${ }^{6}$ Una alternativa es considerar como unidad de análisis al hogar, en cuyo caso cada hogar es representado una sola vez en las estadísticas, independientemente de su tamaño. La opción seguida en el texto es recomendada por los expertos, dado que el referente final del bienestar son los individuos (Deaton, 1997; De Ferrantis et al., 2003). En cualquier caso las conclusiones del texto no dependen de esta opción metodológica.
} 
Todos los indicadores son coincidentes para señalar que la desigualdad de ingresos se mantuvo relativamente estable entre 1990 y 2000, para luego caer en el período 2000 a 2006. Es importante notar que la reducción de la desigualdad no está acotada al año 2006, sino que ya estaba presente en el año 2003, lo cual establece una posible tendencia antes que un efecto específico a un determinado año.

Una mirada más detallada del cambio en la distribución de ingreso se realiza a través de la tasa de crecimiento del ingreso per cápita del hogar a nivel de los distintos deciles (Cuadro $\mathrm{N}^{\circ}$ 2). En el período 1990-2000 el crecimiento del ingreso es relativamente parejo entre los deciles, de modo que la distancia relativa entre los ingresos tiende a mantenerse y los indicadores de desigualdad permanecen estables. Esto último puesto que las medidas de desigualdad son invariantes a la escala; de manera que no cambian si todos los ingresos crecen en igual proporción ${ }^{7}$.

En cambio, durante el período 2000 a 2006 el crecimiento del ingreso presenta una clara tendencia decreciente a lo largo de la distribución. Así, los deciles más bajos experimentan incrementos (acumulados) del ingreso medio por arriba del 20\%, los deciles medio-altos crecen en el entorno del

\begin{tabular}{lccc} 
CUADRO No 2: & \multicolumn{2}{l}{ TASAS DE CRECIMIENTO DEL INGRESO PER CÁPITA POR DECIL DE } \\
& & & \\
\hline HOGARES (\%) & & \\
\hline & $2000-03$ & $2003-06$ & \\
\hline 1 & 10,2 & 22,2 & 34,6 \\
2 & 6,5 & 17,5 & 25,1 \\
3 & 5,7 & 16,4 & 23,1 \\
4 & 4,7 & 15,7 & 21,1 \\
5 & 3,3 & 14,7 & 18,5 \\
6 & 3,3 & 13,5 & 17,3 \\
7 & 2,6 & 12,7 & 14,3 \\
8 & 1,8 & 12,3 & 0,3 \\
9 & 0,2 & 11,2 & 9,2 \\
10 & 0,1 & 0,6 & 9,2 \\
Promedio & 1,5 & 7,6 & \\
\end{tabular}

Fuente: Cálculos basados en encuestas Casen, años respectivos.

${ }^{7}$ Esto es, si I(x) es la función que relaciona el vector $\mathrm{x}$ de ingresos con el indicador I; entonces $\mathrm{I}(\mathrm{x})=\mathrm{I}(\mathrm{ax})$, donde a es una constante positiva (Cowell, 2000). Esta propiedad es cuestionada en estudios de percepción de la desigualdad, puesto que la mayor parte de los entrevistados señala que un incremento en las distancias absolutas de ingresos implican un aumento en la desigualdad, aun cuando no haya habido cambio en las distancias relativas (Amiel y Cowell, 1999). 
$10 \%$, mientras que el decil más rico presenta un crecimiento del ingreso cercano a cero.

Estos datos ratifican la tendencia señalada previamente: la caída en la desigualdad no es privativa del año 2006, sino que se inicia en el período 2000-2003 para luego profundizarse entre 2003 y 2006. Hay, eso sí, un dato fuera de tendencia, cual es la abrupta baja en el crecimiento del ingreso del decil 10 en el año 2006. Ésta se relaciona con una reducción significativa en el ingreso de los empleadores, la cual requiere ser examinada en mayor grado de detalle ${ }^{8}$.

Un punto importante a destacar es la relación entre el crecimiento del ingreso por deciles y el crecimiento del ingreso promedio o conjunto. El Cuadro $\mathrm{N}^{\circ} 2$ muestra que el crecimiento del ingreso de los deciles 1 a 9 está por arriba del crecimiento del ingreso del conjunto; lo cual podría parecer contradictorio pues este último debiera ser representativo de las partes. Sin embargo, el crecimiento del ingreso del conjunto se calcula como un promedio ponderado del crecimiento del ingreso de cada decil, donde la ponderación es la participación de cada decil en el ingreso total. El gran peso que tiene el decil 10 en el total de ingresos (42\% en el año 2006) explica la divergencia entre el resultado de las partes versus el resultado del conjunto ${ }^{9}$.

Se desprende de lo anterior que la evaluación del comportamiento de la economía a través del crecimiento del ingreso promedio, que es una práctica habitual, puede ser poco informativo del crecimiento que experimenta el ingreso de grupos mayoritarios de la población. Esto sucede precisamente en el período 2000-2006, cuando el crecimiento del ingreso medio subestima el crecimiento medio de los deciles 1 a 9. La conclusión contraria se aplicaría si el ingreso del decil rico exhibiese una tasa de crecimiento más alta que el ingreso del resto de los deciles.

El Gráfico $\mathrm{N}^{\circ} 1$ presenta el crecimiento del ingreso per cápita de los hogares a nivel de centiles de la distribución de la variable entre 2000 y 2006. Ello entrega información más detallada de la dinámica distributiva. Los datos muestran la existencia de un patrón sorprendentemente continuo del cambio en el ingreso, puesto que cada centil de la distribución presenta un

\footnotetext{
${ }^{8}$ La participación de los empleadores en el ingreso total cae de 14,3\% a 10,6\% entre 2003 y 2006, mientras que la participación del ingreso por cuenta propia sube de $16,6 \%$ a $19,3 \%$ en igual período de tiempo. Es posible que haya habido algún cambio de clasificación entre los ingresos por cuenta propia y los ingresos de los empleadores, que contribuya a explicar tal situación.

${ }^{9}$ Sea $\rho$ la tasa de crecimiento del ingreso promedio y $\rho$ i la tasa de crecimiento del ingreso del decil i; entonces $\rho=\Sigma \lambda i$ i; donde $\lambda i$ es la participación del ingreso del decil i en el ingreso total.
} 
GRÁFICO No 1: TASA DE CRECIMIENTO DEL INGRESO PER CÁPITA 2000 A 2006

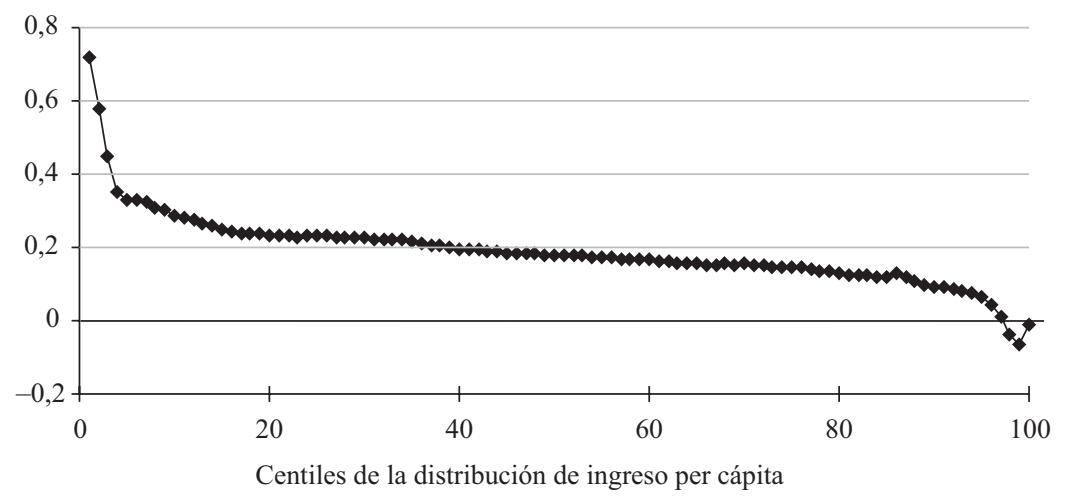

incremento de ingreso algo inferior respecto del centil precedente. Este tipo de resultados es muy poco frecuente, puesto que típicamente existe una dosis elevada de "ruido estadístico" que origina relaciones gráficas más irregulares.

La reducción de la desigualdad a partir del año 2000 es un resultado importante, puesto que representa un punto de inflexión respecto del nivel alto y estable que presentaba previamente la desigualdad. Sin embargo, el nivel de desigualdad sigue siendo muy elevado y se está lejos de los estándares que caracterizan a los países desarrollados. En términos del coeficiente de Gini, éste se reduce en 3,6 puntos en el período reciente, un resultado significativo pero que representa menos del $15 \%$ de la brecha existente respecto de países desarrollados.

La interpretación de los resultados anteriores debe tener presente lo establecido en el ejemplo de la Tabla $\mathrm{N}^{\circ} 1$. Un crecimiento del ingreso del decil 1 por arriba del decil 2 no debe ser interpretado como que el ingreso de los hogares que pertenecían al decil 1 en el año inicial creció más rápidamente. La interpretación correcta es que el ingreso del decil 1 creció más rápido, cualesquiera que sean los hogares que integren ese decil en cada año.

Curvas de Lorenz

La curva de Lorenz es una representación gráfica de la distribución de ingresos, la cual resulta particularmente útil para realizar análisis comparativos de la desigualdad en el tiempo. La curva de Lorenz relaciona la distribución acumulada de hogares con su respectiva participación en el 
total de ingresos. Una distribución igualitaria de ingresos tiene asociada una curva de Lorenz con forma de línea recta o de 45 grados; puesto que el $10 \%$ más pobre de los hogares obtiene el 10\% del total de los ingresos; el $20 \%$ más pobre tiene el $20 \%$ del total de ingresos, etc. ${ }^{10}$.

El gráfico A-1 en el Anexo presenta la curva de Lorenz de la distribución de ingresos de 2006. La curva de Lorenz está por debajo de la línea de los 45 grados, representando el caso de una distribución desigual, puesto que el $10 \%$ más pobre de los hogares obtiene sólo el 1,5\% del total de los ingresos, el $20 \%$ más pobre obtiene el $4,1 \%$, etc.

La comparación de las curvas de Lorenz de distribuciones del ingreso en distintos períodos de tiempo informa sobre las tendencias de la desigualdad. Una distribución es menos desigual que otra si la curva de Lorenz de la primera está por sobre la curva de Lorenz de la segunda; esto es, más cercana a la línea de los 45 grados o distribución igualitaria.

El Gráfico N ${ }^{0} 2$ presenta la distancia entre las curvas de Lorenz del año 2006 respecto de años anteriores. En cada caso esta distancia es positiva para todos los valores posibles de la curva de Lorenz, de modo que existe menor desigualdad de ingresos en 2006 respecto de los años previos,

GRÁFICO N 2: $\quad$ DISTANCIA ENTRE CURVAS DE LORENZ (BASE 2006)

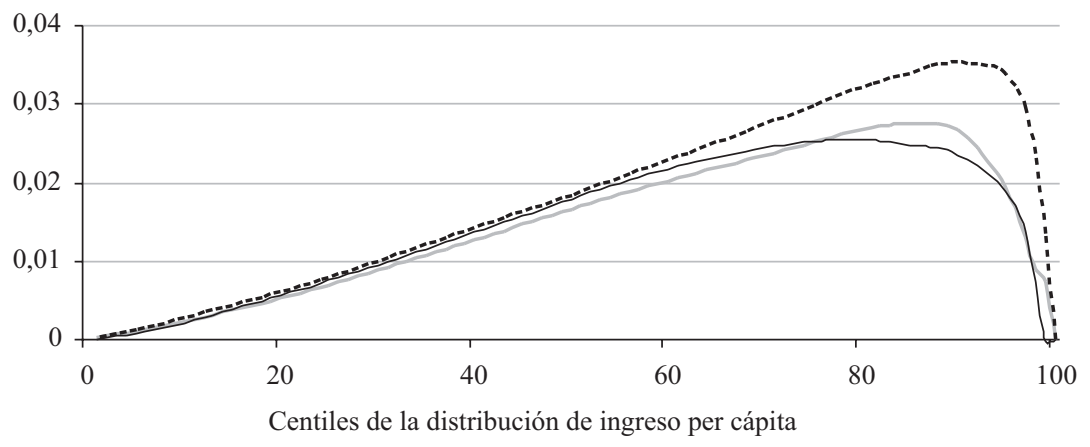

$1990-2006$

-1996-2006 - - - 2000-2006

\footnotetext{
${ }^{10}$ La distribución acumulada de hogares resulta de ordenar a los hogares de menor a mayor ingreso; de modo que p es el porcentaje de hogares con ingresos menor igual a un determinado monto, y L(p) es el porcentaje del ingreso total que tienen estos hogares. La curva de Lorenz relaciona p y L(p); la distribución igualitaria de ingresos implica que $\mathrm{p}=$ $\mathrm{L}(\mathrm{p})$, mientras que una distribución desigual es caracterizada por $\mathrm{p}<\mathrm{L}(\mathrm{p})$.
} 
en todo el rango de la distribución de ingresos. El resultado descrito se denomina dominancia de la curva de Lorenz y no ocurre frecuentemente. Es más típico que las curvas de Lorenz de diferentes períodos se crucen entre sí, originando rankings ambiguos de desigualdad en el tiempo.

Las curvas de Lorenz, al igual que los demás indicadores de desigualdad, son informativos de la distribución de los ingresos pero no así de su nivel medio. Podría ocurrir que existiera dominancia de Lorenz, pero a un nivel de ingreso medio más bajo; en cuyo caso el país sería más igualitario pero más pobre. El resultado es ambiguo en términos de bienestar social. En cambio, cuando la dominancia de Lorenz es acompañada por mayores niveles de ingreso medio es posible concluir que el bienestar social ha aumentado: el país es a la vez más igualitario y más rico. Éste es el caso del año 2006, que domina tanto en Lorenz como en el nivel del ingreso medio a los años anteriores ${ }^{11}$.

\section{La pobreza}

Esta sección evalúa la trayectoria de la pobreza en el período 19902006. Su inclusión en un artículo que tiene por eje a la desigualdad de ingresos responde a la relación existente entre ambas variables, puesto que la desigualdad y la pobreza se derivan de un mismo sustrato. Mientras la desigualdad trata sobre las distancias de los ingresos a lo largo de la distribución, la pobreza corresponde a la parte inferior de la distribución, aquella que está por debajo de la línea de pobreza ${ }^{12}$.

En el Cuadro $\mathrm{N}^{\circ} 3$ se presenta un conjunto de indicadores para caracterizar la evolución de la pobreza en el tiempo. Estos son: (i) el porcentaje de pobres, que son quienes viven en hogares cuyo ingreso per cápita es menor a la línea de pobreza; (ii) el porcentaje de extrema pobreza, cuyo ingreso del hogar es inferior a la línea de indigencia; (iii) el porcentaje del PIB que habría que destinar a los hogares pobres para que alcanzaran la línea de pobreza.

Los dos primeros indicadores son medidas de la incidencia de la pobreza; esto es, informan respecto de cuántas personas son pobres. El

${ }^{11}$ El resultado de bienestar se establece en Atkinson (1970) y se basa en una función de bienestar que sea una función creciente y cóncava de los ingresos individuales. Esta función es anónima, puesto que sólo utiliza información de ingresos y no así las identidades de los hogares subyacentes. De modo que puede haber aumentos de bienestar social junto a pérdidas de ingresos a nivel de casos individuales.

${ }^{12}$ La discusión que sigue está referida a la pobreza como insuficiencia de ingresos, que es la definición tradicional utilizada para medir la pobreza. En Larrañaga (2007b) se discute la medición de la pobreza en dimensiones adicionales al ingreso. 
CUADRO No 3: $\quad$ INDICADORES DE POBREZA, 1990-2006

\begin{tabular}{lrrr}
\hline \% de pobres & \% extrema pobreza & $\begin{array}{r}\text { Brecha de pobreza } \\
\text { como \% PIB }\end{array}$ \\
\hline 1990 & 38,4 & 12,8 & 4,09 \\
1996 & 23,1 & 5,8 & 1,36 \\
2000 & 20,5 & 5,7 & 1,20 \\
2003 & 18,7 & 4,7 & 0,95 \\
2006 & 13,7 & 3,2 & 0,49 \\
\hline
\end{tabular}

Fuente: Cálculos basados en encuestas Casen, años respectivos.

tercer indicador es una medida de incidencia, pero también de profundidad de pobreza. Puede ser interpretado como el gasto necesario para "eliminar" la pobreza si se conociera con exactitud el ingreso de cada hogar y si este se mantuviera fijo en presencia de la transferencia ${ }^{13}$. Se trata de una cota inferior puesto que el costo efectivo de tal política debe considerar que hay costos adicionales de focalización e incentivos. Ello, puesto que hay incentivos a subdeclarar el ingreso si tal información va a ser utilizada para calcular una transferencia que crece mientras menor sea el ingreso declarado. Además, la existencia de tal transferencia reduce el ingreso autónomo si se produce algún tipo de desincentivo al trabajo.

La reducción de la pobreza en el período 1990 a 2006 es un resultado claro y significativo, más allá del indicador que se utilice para medir la variable. Así, el porcentaje de pobres en el año 2006 es un 36\% del nivel en 1990; mientras que el índice de indigencia en 2006 representa sólo la cuarta parte de su nivel inicial. Por su parte, en el año 1990 se requería transferir un 4,1\% del PIB como cota mínima para "eliminar" la pobreza; en el año 2006 tal monto ascendía sólo a un $0,49 \%$ del PIB. El último resultado representa una síntesis de la caída en el porcentaje de pobreza, incrementos en el ingreso medio de los pobres y el aumento en el ingreso medio de la economía (véase nota 12).

La caída de la pobreza ocurre en tres fases a lo largo del período: una primera fase de rápida reducción de la pobreza (1990-1996); una segunda

${ }^{13} \mathrm{El}$ costo de reducción de la pobreza es igual a n(z-u)/(Ny); donde $\mathrm{n}$ es el número de pobres, z es la línea de pobreza, u es el ingreso medio de los pobres, (z-u) es la brecha de pobreza, $\mathrm{N}$ es la población del país, y es el producto medio de la economía (de modo que Ny es el PIB). El indicador también puede expresarse como $\mathrm{H}(\mathrm{z}-\mathrm{u}) / \mathrm{y}$; donde $\mathrm{H}$ es el porcentaje de pobreza $(\mathrm{n} / \mathrm{N})$. Un indicador relacionado es la brecha de pobreza, que es igual a $\mathrm{H}(1-\mathrm{u} / \mathrm{z})$. 
etapa de relativo estancamiento (1996-2000) y una tercera fase de recuperación de la tendencia a la baja (2000-2006). En el primer intervalo el porcentaje de pobreza cae a una tasa promedio de 2,56 puntos porcentuales por año, en el segundo período la pobreza cae en 0,75 puntos por año y en el tercer período en 1,08 puntos por año. Las cifras respectivas para la reducción de la indigencia son 1,22, 0,03 y 0,40.

\section{La línea de la pobreza}

No existe un procedimiento estandarizado a nivel internacional para medir la pobreza, a diferencia de lo que ocurre con las cuentas nacionales, las finanzas públicas y otros indicadores económicos. La medición de la pobreza admite variantes que incluyen criterios absolutos, como es el caso de una línea de US\$ 1 o 2 diarios per cápita utilizada por el Banco Mundial, así como criterios relativos, como es una línea del $60 \%$ de la mediana del ingreso utilizada por diversos países europeos. El procedimiento que se elija se relaciona con la interpretación de las "necesidades mínimas", que pueden ser consideradas absolutas o relativas respecto del nivel de vida en la sociedad.

En Chile, así como en la generalidad de los países de América Latina, la pobreza se mide a través del método de la canasta básica de alimentos. Éste tiene dos componentes. Primero, el costo de adquisición de una canasta de alimentos propiamente tal, que satisfaga los requerimientos nutricionales de los miembros de un hogar, y que define la línea de indigencia o extrema pobreza. Segundo, un factor de ajuste para incluir el gasto en otras necesidades: vestuario, transporte, vivienda, etc. En ambos casos se trabaja sobre la base del gasto de consumo medido en encuestas de presupuestos familiares, considerando como referencia a hogares de estratos medios.

El método de la canasta básica considera elementos absolutos (requerimientos nutricionales), pero también incluye un componente relativo puesto que se basa en el consumo efectivo que realiza el estrato de hogares de menor nivel de ingreso que cumple los requerimientos calóricos, el cual se modifica en el tiempo según cambien el nivel de ingresos y los patrones de consumo. En cambio, la variante europea que privilegia el factor relativo tiende a confundirse con una medida puramente distributiva, para la cual se dispone de otro tipo de indicadores. De hecho, su aplicación en países en desarrollo podría inducir resultados contradictorios, como que un país pobre (bajo ingreso per cápita) no contenga población pobre en su interior (si todos están por arriba del $60 \%$ de la mediana del ingreso). 
La línea de pobreza utilizada en el país está basada en la encuesta de presupuestos del año 1986. La línea se ha reajustado según el índice de precios del consumidor, para mantener su valor en términos reales, pero no ha sido ajustada por los cambios en la estructura de consumo que han ocurrido desde la citada fecha. Para tal efecto se dispondría de la encuesta de presupuesto de 1996 y, próximamente, de la encuesta del año 2007.

El efecto que tendrían líneas alternativas sobre la medición de la pobreza puede ser conocido a través de micro-simulaciones en las bases de datos Casen. A tal efecto, el Cuadro $\mathrm{N}^{\circ} 4$ presenta los resultados de tales estimaciones para líneas de pobreza que fluctúan entre M\$ 25,0 y M\$ 75,0 (\$ de 2006). La referencia es una línea de M\$ 45,0, que aproxima el valor efectivamente utilizado ${ }^{14}$.

Hay dos resultados de particular interés que se derivan del ejercicio: el impacto sobre los niveles de pobreza y el efecto sobre la evolución de la pobreza en el tiempo.

El impacto sobre el nivel de pobreza es significativo. Considerando como referencia el año 2006, resulta que un incremento de $\mathrm{M} \$ 45,0$ a M\$ 55,0 de la línea de pobreza aumentaría el porcentaje de pobreza en 14,3\% a 21,3\%, mientras que un incremento de la línea a M\$ 65,0 prácticamente duplicaría el porcentaje de pobreza (de 14,3\% a 28,0\%).

La elevada sensibilidad de la medida de pobreza respecto del valor de la línea se debe a que hay muchos hogares cuyo ingreso está en el entorno de la línea de pobreza. De este modo, cambios en la línea tiene un alto impacto en el porcentaje de pobreza. Este resultado sugiere que hay que tener mayor cuidado en la interpretación de las estadísticas de pobreza en el país, considerando que hay dudas razonables respecto del verdadero valor de la línea de pobreza.

CUADRO No 4: PORCENTAJE DE POBREZA PARA LÍNEAS ALTERNATIVAS DE POBREZA

\begin{tabular}{lrrrrrr}
\hline \multicolumn{5}{c}{ Línea de pobreza (en M\$ 2006) } \\
\cline { 2 - 6 } & 25,0 & 35,0 & 45,0 & 55,0 & 65,0 & 75,0 \\
\cline { 2 - 6 } 1990 & 15,2 & 27,6 & 38,4 & 47,6 & 55,2 & 61,2 \\
1996 & 7,1 & 13,7 & 21,6 & 29,7 & 36,7 & 43,0 \\
2000 & 7,9 & 16,2 & 24,7 & 32,8 & 40,9 & 47,0 \\
2003 & 5,9 & 11,9 & 19,6 & 27,5 & 34,8 & 41,4 \\
2006 & 4,1 & 8,1 & 14,3 & 21,3 & 28,0 & 34,5 \\
\hline
\end{tabular}

Fuente: Cálculos basados en encuestas Casen, años respectivos

${ }^{14}$ Considerando un promedio ponderado de los componentes urbano y rural. 
El segundo resultado de interés es la relación entre el valor de la línea y la tendencia de la pobreza en el tiempo. La tendencia es claramente descendente, con independencia de la línea que se utilice. Sin embargo, la gradiente de descenso no es constante, sino que decrece cuando aumenta el valor de la línea. Bajo el umbral mínimo (M\$25,0) se tiene que la pobreza en el año 2006 representa casi una cuarta parte de la pobreza en 1990 (15,2\% a 4,1\%). Bajo el umbral máximo (M\$ 75,0) la caída de la pobreza es sólo a un 55\% del valor inicial (de 61,2\% a 34,5\%).

El resultado está nuevamente relacionado con el porcentaje de hogares cuyo ingreso está en el entorno de la línea. Mientras más alta es la línea de pobreza, menos densa es la función de ingresos en su entorno, y menor es el efecto de los cambios de la distribución que ocurren en el tiempo sobre el porcentaje de pobreza.

\section{Efectos de crecimiento y distribución}

La distribución de ingresos cambia en el tiempo, tanto porque se modifica el ingreso medio (desplazamientos paralelos de la distribución) como porque cambia la desigualdad (forma de la distribución). Dado que la pobreza corresponde al área inferior de la distribución del ingreso, entonces los cambios en pobreza pueden ser "explicados" en términos de cambios en el nivel medio y en la forma de la distribución. Estos son los citados efectos crecimiento y distribución, también denominados descomposición de DattRavallion ${ }^{15}$.

Específicamente, el efecto crecimiento es el cambio en la pobreza producto del aumento (disminución) del ingreso promedio, suponiendo constante la desigualdad de los ingresos; mientras que el efecto distribución es el cambio en la pobreza originado en el cambio en la desigualdad, asumiendo constante el nivel del ingreso medio entre ambos períodos ${ }^{16}$.

La descomposición de Datt-Ravallion se realiza para el porcentaje de pobreza y la brecha de pobreza (véase nota 12), para cada uno de los subperíodos principales de análisis. Los resultados se presentan en el Cuadro $\mathrm{N}^{\circ} 5$.

${ }^{15}$ El nombre apropiado es efecto desigualdad antes que efecto distribución, puesto que la distribución incluye desplazamientos paralelos (crecimiento). Sin embargo en el texto se mantiene la denominación habitual de efectos crecimiento y distribución.

${ }^{16} \mathrm{El}$ cálculo de los citados efectos requiere micro-simular la medida de pobreza utilizando una estimación econométrica de la distribución de los ingresos. Hay distintas especificaciones funcionales para tal propósito, utilizándose en este caso la propuesta por Kakwani. El detalle de la metodología aparece en Datt y Ravallion (1983); la primera aplicación en Chile corresponde a Larrañaga (1994). 
CUADRO N ${ }^{\circ}$ 5: $\quad$ DESCOMPOSICIÓN DE DATT-RAVALLION

\begin{tabular}{lcccc}
\hline Período & $\begin{array}{c}\text { Efecto } \\
\text { crecimiento } \%\end{array}$ & $\begin{array}{c}\text { Efecto } \\
\text { distribución } \%\end{array}$ & Residuo \% & $\begin{array}{c}\text { Caída en } \\
\text { pobreza }\end{array}$ \\
\hline Porcentaje pobres & & & & \\
1990-96 & 95,0 & 9,4 & $-4,4$ & 17,3 \\
$1996-00$ & 82,6 & 20,2 & $-2,7$ & 2,6 \\
$2000-06$ & 21,9 & 75,7 & 2,4 & 6,8 \\
$1990-06$ & 71,7 & 22,9 & 5,5 & 24,7 \\
& & & & \\
Brecha pobreza & & & & 6,5 \\
$1990-96$ & 93,9 & 8,0 & $-2,0$ & 0,9 \\
$1996-00$ & 87,3 & 15,0 & $-2,3$ & 2,8 \\
$2000-06$ & 22,8 & 81,3 & $-4,1$ & 7.7 \\
$1990-06$ & 72,6 & 35,5 & $-8,1$ & \\
\hline
\end{tabular}

El efecto crecimiento es dominante en el período 1990-1996, explicando alrededor del 95\% de la reducción en la pobreza en cualquiera de sus mediciones. En cambio, entre los años 2000 y 2006 se revierte la importancia de los efectos y es ahora el cambio en la distribución que explica cerca del 80\% de la reducción de la pobreza en el período.

Los efectos crecimiento y distribución están expresados en términos porcentuales; para conocer su magnitud absoluta se requiere multiplicar los citados porcentajes por la reducción en el indicador de pobreza. De hecho, en el período intermedio (1996-2000) el efecto crecimiento es dominante, pero la reducción de la pobreza es baja en términos absolutos.

Los resultados descritos son plenamente consistentes con los patrones de crecimiento y desigualdad que caracterizan a la distribución de ingresos por deciles (Cuadro No 2). En el período 1990-1996 el crecimiento del ingreso es fuerte y parejo a través de los deciles, de modo que la distribución se mantiene constante. Es evidente por tanto que el cambio en la pobreza en este período tiene que deberse exclusivamente al efecto crecimiento. En cambio, en el período 2000-2006 el crecimiento de los ingresos favorece a los ingresos más bajos, induciendo una caída de la desigualdad. La reducción asociada de la pobreza se asocia por tanto a un efecto distribución.

La descomposición de Datt-Ravallion no informa sobre los factores subyacentes a los efectos crecimiento y distribución. En la sección siguiente se muestra que los cambios distributivos de ingresos entre 2000-2006 están fuertemente influenciados por la distribución de los salarios. Por tan- 
to, los cambios en pobreza están relacionados con dinámicas que se producen en el mercado laboral.

Una materia distinta es si atribuir la dinámica laboral a la política social o a los mecanismos de mercado propiamente tales. En principio, las variables económicas responden tanto a factores de política como de mercado. Por ejemplo, un incremento en los salarios puede ser originado en una mayor demanda de empleos que responda a mejores expectativas de ventas de las empresas. Sin embargo, los incrementos salariales también dependerán de los niveles de capital humano de los trabajadores, los cuales están estrechamente relacionados con la política social puesto que la mayor parte de la población accede a educación y salud financiada con recursos públicos. La estimación de este tipo de efectos excede por lejos los alcances del presente artículo.

\section{Dominancia}

El análisis de dominancia (Atkinson, 1987) señala que la reducción de la pobreza entre dos años cualquiera es robusta a la elección de la línea de pobreza y del indicador de pobreza si la función de distribución de ingresos del año final es menor a la función del año inicial (en el rango de líneas de pobreza elegido).

Un resultado similar rige para funciones de pobreza cóncavas en ingreso (FGT 2), que sean sensibles a la distribución al interior de la pobreza. Esta vez se requiere dominancia a nivel de las funciones acumuladas de distribución.

Los Gráficos No A-2 a A-4 en el Anexo presentan el resultado de dominancia, utilizando gráficos de las funciones de distribución del ingreso en el año 2006 y anteriores. Se muestra que el resultado de dominancia rige para el año 2006, tanto a nivel de orden 1 y 2. De esta manera, podemos establecer que la reducción de la pobreza en el período es un resultado robusto, que generaliza para cualquier valor que se utilice para la línea de pobreza, así como para cualquier indicador que se elija para medir la pobreza.

\section{El rol de los salarios en el cambio de la desigualdad}

Los salarios tienen un rol protagónico en la reciente caída de la desigualdad de ingresos. Ello ocurre por la confluencia de dos factores: (i) la elevada participación de la partida de salarios en el ingreso del hogar; (ii) la compresión de las brechas salariales en el período reciente. Ambos facto- 
res deben estar presentes para que los salarios hayan tenido el rol protagónico detrás de la reducción de la desigualdad. El primer factor asegura que cualquier cambio en los salarios tendrá un impacto significativo sobre los ingresos del hogar; el segundo factor estipula que los salarios cambiaron en la dirección necesaria para reducir la desigualdad de los ingresos en el período.

Esta sección documenta el rol de los salarios a través de una descomposición de los cambios en la desigualdad medida a través del coeficiente de Gini; analiza la distribución de los salarios y caracteriza sus recientes cambios, postulando algunas hipótesis que deben ser exploradas en estudios futuros, y finalmente hace referencia al rol desempeñado por el empleo en la dinámica distributiva.

Descomposición del coeficiente de Gini

El rol que cumplen los salarios en los cambios distributivos puede ser evaluado a través de una metodología de descomposición desarrollada en Shorrocks (1983). Ésta se basa en la desagregación del coeficiente de Gini del ingreso total en términos de las $K$ partidas de ingresos que componen el ingreso total.

$$
G=\sum_{k} C_{k} \Psi_{k}=C_{1} \Psi_{1}+C_{2} \Psi_{2}+\ldots \ldots \ldots+C_{k} \Psi_{k}
$$

Esta expresión muestra que el coeficiente de Gini $(G)$ depende del coeficiente de concentración $\left(\mathrm{C}_{\mathrm{k}}\right)$ de las distintas partidas de ingresos y del peso que éstas tienen en el ingreso total $\left(\Psi_{k}\right)$. El coeficiente de concentración es una variante del coeficiente de Gini, puesto que es un promedio ponderado de los ingresos $\mathrm{k}$, utilizando como ponderadores el orden que cada hogar ocupa en la distribución del ingreso total ${ }^{17}$.

El coeficiente de concentración mide la distribución del ingreso k entre los hogares, en relación a la distribución del ingreso total. Si el ingreso $\mathrm{k}$ se distribuye en forma proporcional al ingreso total, el coeficiente de correlación del ingreso k es igual al coeficiente de Gini del ingreso total; si el ingreso k se distribuye en forma más (menos) regresiva que el ingreso total,

\footnotetext{
${ }^{17}$ En cambio, el coeficiente de Gini ocupa como ponderador el orden de la distribución del mismo tipo de ingreso. El coeficiente de concentración también puede calcularse como uno menos dos veces el área bajo la curva de concentración; esta última relaciona la distribución acumulada de hogares según el ingreso total con la distribución acumulada de ingresos $\mathrm{k}$.
} 
el coeficiente de correlación respectivo es mayor (menor) que el coeficiente de Gini.

Considerando las diferencias en el tiempo $(\Delta)$ en la expresión anterior, se obtiene:

$$
\Delta G=\sum_{k}\left(C_{k} \Delta \Psi_{k}+\Delta C_{k} \Psi_{k}\right)
$$

El primer término en la sumatoria representa el efecto de los cambios en la participación, mientras que el segundo término corresponde al efecto del cambio en el coeficiente de concentración. Ya que la sumatoria de los cambios en los pesos de cada partida de ingreso es cero, es posible adicionar a la expresión anterior el producto de esta sumatoria por el Gini promedio, reordenar términos y obtener:

$$
\Delta G=\sum_{k}\left(\left(C_{k}-\bar{G}\right) \Delta \Psi_{k}+\Delta C_{k} \bar{\Psi}_{k}\right)
$$

La expresión final señala que el cambio en el coeficiente de Gini puede expresarse en términos de dos componentes: (i) efecto participación, que es igual a los cambios en la participación de cada partida de ingresos $\mathrm{k}$ (ponderada por la diferencia entre el coeficiente de concentración del ingreso k y el coeficiente de Gini); (ii) efecto concentración, que es igual a los cambios en el coeficiente de concentración de cada ingreso k (ponderada por la respectiva participación de $\mathrm{k}$ en el ingreso total).

A continuación se presenta una estimación de la contribución que tuvieron las diversas partidas de ingreso en la reducción de la desigualdad en el período 2000-2006. El ejercicio se realiza a nivel del ingreso monetario de los hogares, el cual se divide en cinco componentes: ingresos asalariados; ingresos del trabajo independiente (cuenta propia y empleadores); pensiones (vejez, invalidez, montepíos y orfandad); subsidios monetarios (pensiones asistenciales, subsidio único familiar, bonos del sistema de protección social, etc.), y otros ingresos.

El Cuadro $\mathrm{N}^{\circ} 6$ muestra las partidas de ingresos consideradas en este análisis, la participación de cada una de ellas en el ingreso monetario total y el índice de concentración asociado a cada una de estas partidas. Nótese que el coeficiente de Gini del ingreso monetario es algo distinto del coeficiente asociado al ingreso total per cápita (sección 1), aun cuando ambos reportan igual resultado distributivo.

Durante el período disminuye la concentración de todas las partidas de ingresos. De modo que se espera que el efecto concentración sea impor- 
CUADRO N 6: $^{\circ}$ TASA DE PARTICIPACIÓN Y COEFICIENTE DE CONCENTRACIÓN DE LAS PARTIDAS DE INGRESO MONETARIO

\begin{tabular}{|c|c|c|c|c|}
\hline \multirow[t]{2}{*}{ Partida de ingresos } & \multicolumn{2}{|c|}{ Participación en total (\%) } & \multicolumn{2}{|c|}{ Coeficiente de concentración (\%) } \\
\hline & 2000 & 2006 & 2000 & 2006 \\
\hline Salarios & 45,8 & 47,1 & 47,3 & 42,4 \\
\hline Independientes & 29,2 & 27,3 & 73,2 & 70,9 \\
\hline Pensiones & 7,9 & 6,0 & 37,5 & 26,0 \\
\hline Subsidios & 1,0 & 1,2 & $-23,0$ & $-23,6$ \\
\hline Otros & 16,0 & 18,4 & 65,0 & 59,2 \\
\hline Total & 100,0 & 100,0 & 56,2 & 51,5 \\
\hline
\end{tabular}

Nota: el coeficiente de concentración en el caso del ingreso total es igual al Gini.

Fuente: Calculado sobre la base de encuestas Casen, años respectivos.

tante en la caída de la desigualdad en el período. En relación con la participación de cada partida de ingreso en el ingreso total, los cambios son más pequeños y pueden tener efecto en ambos sentidos en la desigualdad del ingreso total.

El Cuadro $\mathrm{N}^{\circ} 7$ presenta la descomposición del cambio que experimentó la desigualdad entre los años 2000 y 2006, diferenciando el aporte que tuvo cada partida a la reducción de la desigualdad en los efectos concentración y participación.

El resultado más importante es la caída en la concentración de los ingresos asalariados, que por sí solo explica prácticamente la mitad de la reducción en la desigualdad. Esto es, un incremento relativo en los ingresos salariales de los hogares de menor ingreso per cápita, junto a una elevada participación de los ingresos salariales en el ingreso total son responsables de una parte sustantiva de los cambios distributivos en el período 2000 a 2006.

CUADRO No 7: DESCOMPOSICIÓN DEL CAMBIO EN LA DESIGUALDAD DEL INGRESO MONETARIO 2000-2006

\begin{tabular}{lcccc}
\hline Partida de ingresos & $\begin{array}{c}\text { Efecto } \\
\text { participación }\end{array}$ & $\begin{array}{c}\text { Efecto } \\
\text { concentración }\end{array}$ & Efecto total & $\begin{array}{c}\text { Efecto total } \\
(\%)\end{array}$ \\
\hline Salarios & $-0,11$ & $-2,27$ & $-2,38$ & 50,9 \\
Independientes & $-0,34$ & $-0,66$ & $-0,99$ & 21,2 \\
Pensiones & 0,43 & $-0,80$ & $-0,36$ & 7,8 \\
Subsidios & $-0,13$ & $-0,01$ & $-0,13$ & 2,8 \\
Otros & 0,20 & $-1,01$ & $-0,81$ & 17,3 \\
Total & 0,05 & $-4,74$ & $-4,69$ & 100,0 \\
\hline
\end{tabular}


Todas las demás partidas de ingreso presentan una contribución positiva a la caída de la desigualdad a partir de una reducción en su coeficiente de concentración. Sin embargo, su impacto relativo es algo menor, sea porque su participación en el ingreso total es más reducida o porque la variación del coeficiente de concentración es pequeña.

El ingreso del trabajo independiente contribuye en cerca de un 20\% a la caída de la desigualdad. En este caso el efecto participación desempeña un rol también relevante; puesto que esta partida de ingreso presenta un descenso de alrededor de dos puntos en su participación en el ingreso total, induciendo una baja en la desigualdad total dado que se trata de un ingreso con fuerte concentración. Los cambios descritos son atribuibles a una caída en la tasa de participación y en el coeficiente de concentración del ingreso de los empleadores, los cuales son sólo parcialmente compensados por efectos en sentido contrario a nivel del ingreso de los trabajadores por cuenta propia ${ }^{18}$.

La contribución del ingreso de las pensiones a la caída de la desigualdad es relativamente menor (8\%). Sin embargo, tal resultado es producto de efectos concentración y participación que operan en direcciones opuestas. El ingreso de las pensiones se hace menos concentrado y menos importante en el ingreso total a lo largo del período, induciendo un efecto concentración que reduce la desigualdad y un efecto participación que la incrementa (cae la participación de una partida con menor concentración). Como se documenta en la siguiente sección del artículo, tales desarrollos están más relacionados con una reasignación de los adultos mayores hacia la parte inferior de la distribución que a una reducción en la desigualdad de los pagos de pensiones propiamente tal.

Finalmente, los subsidios monetarios contribuyen sólo en un $4 \%$ a la caída de la desigualdad. En este caso, es el efecto participación el que contribuye en mayor medida al cambio que experimenta la desigualdad. La baja incidencia de los subsidios para afectar la desigualdad se origina en su muy baja importancia en el total de ingresos (alrededor del 1\% del total). Nótese en todo caso que el coeficiente de concentración de los subsidios es negativo, indicando que se trata de una partida de ingresos que se concentra en los hogares de bajos ingreso per cápita.

La distribución de los salarios

Los ingresos salariales es un agregado que depende de los salarios y del empleo asalariado. De manera que el cambio en la partida puede deber-

\footnotetext{
${ }^{18}$ Véase nota 7 , supra.
} 
se a cambios a nivel de cualquiera de sus componentes. A continuación se analiza la contribución de los salarios, para luego examinar la variable empleo.

El Cuadro $N^{\circ} 8$ presenta la evolución de la distribución de los salarios en el período 1990 a 2006, considerando sólo a los ocupados con 30 y más horas de trabajo semanal. Al igual que en el caso del ingreso del hogar, la desigualdad salarial se mantiene estable en la década del 90 para caer en las mediciones más recientes. En particular, el coeficiente de Gini de los salarios desciende 3,5 puntos entre 2000 y 2006, que es similar a la caída del Gini del ingreso del hogar.

La caída en la desigualdad de los salarios puede también examinarse a través de su tasa de crecimiento a nivel de centiles de su distribución entre 2000 y 2006. El Gráfico No A-5 en el Anexo muestra que la caída de la desigualdad de los salarios se asocia a un patrón de crecimiento descendente a lo largo de la distribución. Así, los salarios bajos exhiben aumentos cercanos a 15\%, los salarios de nivel medio presentan tasas de crecimiento más moderadas, y los salarios altos tienen crecimientos negativos.

El impacto de los cambios salariales sobre la distribución de ingresos puede examinarse directamente a través del crecimiento del salario a nivel de centiles de la distribución del ingreso per cápita. Esto es, para cada centil de ingreso per cápita se computa el salario medio y se compara su nivel entre los años 2000 y 2006. Los resultados muestran que hay una estrecha relación entre el crecimiento de los salarios y del propio ingreso per cápita (Gráfico No 3 comparado con Gráfico No 1 ).

Todos los elementos descritos apuntan hacia una misma conclusión: la distribución de los salarios habría desempeñado un rol clave en la caída de la desigualdad.

CUADRO No 8: $\quad$ DISTRIBUCIÓN DE LOS INGRESOS DEL TRABAJO Y DE SALARIOS: 19902006 (M\$, 2006)

\begin{tabular}{llllll}
\cline { 2 - 4 } & Año & Media & Mediana & p90/p10 & Gini \\
\cline { 2 - 5 } & 1990 & 176,6 & 110,4 & 5,0 & 44,5 \\
1996 & 225,2 & 140,6 & 6,0 & 45,2 \\
2000 & 267,5 & 174,2 & 5,6 & 45,6 \\
2003 & 267,2 & 162,7 & 5,3 & 43,9 \\
\hline
\end{tabular}

Nota: considera ocupados con 30 y más horas semanales de trabajo.

Fuente: Encuestas Casen, años respectivos. 
GRÁFICO N N 3: TASA CRECIMIENTO SALARIOS 2000 A 2006

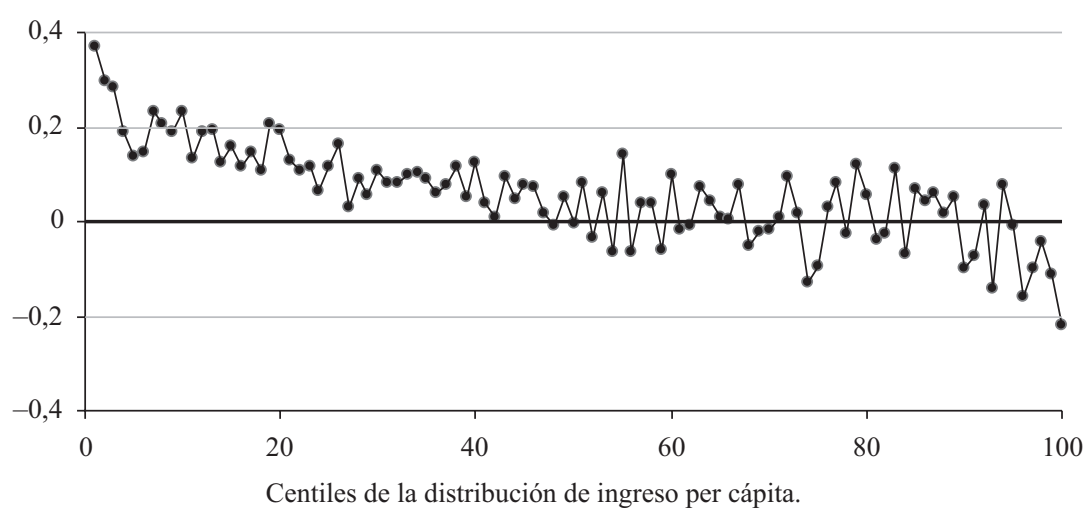

La siguiente etapa de análisis es preguntar por los factores que subyacen detrás de la menor desigualdad de los salarios. A tal respecto, la escolaridad es una variable natural a examinar, puesto que es la determinante principal de los ingresos laborales. Para examinar el punto se clasifica a los ocupados en cinco categorías según años de escolaridad, que corresponden en lo grueso a los principales ciclos de la enseñanza: educación básica o menos (0-8); educación media incompleta (9-11); educación media completa (12); educación terciaria (13-16); educación universitaria completa o postgrados (17 y más). También se distingue entre cohortes de edad, 2544 años y 45-65 años, para identificar posibles efectos relacionados con la experiencia laboral. El análisis excluye a los menores de 25 años, puesto que una parte aún está estudiando.

Las tendencias en la desigualdad salarial se pueden examinar en términos de la comparación de los salarios medios según categorías de educación y de edad. Estos muestran que hay una reducción de la brecha de salarios según nivel educacional: el salario crece más en los grupos con menos escolaridad, al tiempo que decrece en el caso de los grupos con mayor escolaridad. Éste es un resultado que opera a nivel de tendencia en el período 2000 a 2006 (Cuadro No 9).

La caída en el salario medio de los trabajadores con 12 y más años de escolaridad se produce en un período de crecimiento de la economía y del empleo. Una posible explicación de tal desarrollo son los fuertes aumentos en la oferta de trabajadores con estudios secundarios completos, que presionan a la baja los salarios de los ocupados con tal nivel de calificación. En el caso de las personas con estudios de nivel terciario y universitario puede 
CUADRO No 9: $\quad$ ÍNDICE SALARIOS REALES (2000:100)

\begin{tabular}{|c|c|c|c|c|c|c|}
\hline & \multicolumn{6}{|c|}{ Años de escolaridad } \\
\hline & $0-8$ & $9-11$ & 12 & $13-16$ & 17 y más & Todos \\
\hline \multicolumn{7}{|c|}{ Edad: 25-44 } \\
\hline 2000 & 100,0 & 100,0 & 100,0 & 100,0 & 100,0 & 100,0 \\
\hline 2003 & 106,2 & 102,5 & 98,5 & 97,5 & 88,5 & 98,3 \\
\hline 2006 & 113,2 & 109,3 & 98,4 & 101,6 & 89,3 & 100,8 \\
\hline \multicolumn{7}{|c|}{ Edad: 45-65 } \\
\hline 2000 & 100,0 & 100,0 & 100,0 & 100,0 & 100,0 & 100,0 \\
\hline 2003 & 101,9 & 97,7 & 90,7 & 124,8 & 93,0 & 103,5 \\
\hline 2006 & 107,9 & 100,6 & 94,1 & 96,7 & 93,9 & 94,4 \\
\hline
\end{tabular}

Nota: incluye a asalariados que trabajan 30 y más horas semanales.

haber un efecto adicional asociado a una caída en la calidad media de los egresados, habida cuenta de la diversificación de la oferta educacional ${ }^{19}$.

La reducción de la desigualdad salarial no se remite sólo a consideraciones de oferta y demanda según niveles educacionales, sino que también hay factores que operan al interior de los grupos con similar nivel de escolaridad. Esto se muestra en el Cuadro $\mathrm{N}^{\circ} 10$, que presenta la brecha de salarios medida por la relación entre los percentiles 90 y 10 del salario al interior de

CUADRO N 10: BRECHA DE SALARIOS (COEFICIENTES PERCENTIL 90 A PERCENTIL 10)

\begin{tabular}{|c|c|c|c|c|c|c|}
\hline & \multicolumn{6}{|c|}{ Años de escolaridad } \\
\hline & $0-8$ & $9-11$ & 12 & $13-16$ & 17 y más & Todos \\
\hline \multicolumn{7}{|c|}{ Edad: 25-44 } \\
\hline 2000 & 3,00 & 2,97 & 3,57 & 5,00 & 6,32 & 5,62 \\
\hline 2003 & 2,86 & 2,88 & 3,00 & 4,32 & 6,00 & 5,43 \\
\hline 2006 & 2,75 & 2,60 & 3,04 & 4,44 & 6,75 & 4,76 \\
\hline \multicolumn{7}{|c|}{ Edad: 45-65 } \\
\hline 2000 & 3,33 & 4,29 & 4,75 & 5,38 & 6,82 & 6,75 \\
\hline 2003 & 2,50 & 3,46 & 3,92 & 6,15 & 5,56 & 6,67 \\
\hline 2006 & 3,13 & 3,50 & 3,85 & 6,15 & 5,00 & 6,00 \\
\hline
\end{tabular}

Nota: incluye a asalariados que trabajan 30 y más horas semanales.

${ }^{19}$ La caída en la calidad media se originaría en la expansión de oferentes y de demandantes, donde los entrantes en el margen en ambos lados del mercado tienen menor calidad a los inframarginales. 
cada celda educación/edad. En ocho de las diez celdas se constata una apreciable caída de la desigualdad, a nivel de grupos de ocupados con igual nivel educacional y tramo de edad. Por lo general, los cambios referidos operan a nivel de tendencia antes que para un año específico.

\section{Ocupación}

Cambios en los patrones de ocupación pueden causar cambios en la distribución de los ingresos. Por ejemplo, un incremento en la ocupación de personas con bajo nivel de escolaridad inducirá un aumento en el ingreso de hogares de la parte inferior de la distribución, causando una reducción de la desigualdad de los ingresos, otros factores constantes.

El mercado laboral presenta fuerte actividad en el período 2000 a 2006. El conjunto de personas ocupadas acumuló un crecimiento de 23,4\%, según las cifras de las respectivas encuestas Casen. El crecimiento de la ocupación es importante y refleja la recuperación de los niveles de empleo después de la crisis de fines de los 90, así como la absorción de nuevos entrantes en el mercado laboral. El empleo femenino crece más rápidamente que el empleo de los hombres (30,2\% versus 19,5\%), reflejando la incorporación de mujeres de todas las cohortes de edad al mercado laboral, mientras que los nuevos entrantes hombres estarían circunscritos a las cohortes jóvenes.

Estadísticas similares caracterizan el empleo de los asalariados, quienes representan alrededor del $75 \%$ del total de ocupados. Éste es el grupo de mayor interés para nuestros propósitos, dado el rol protagónico de los ingresos salariales en la caída de la desigualdad. A tal respecto, en el período de análisis hay un fuerte crecimiento del empleo en los grupos de escolaridad intermedia, correspondientes a los egresados de la educación media que se expanden en un $41 \%$ (Cuadro $\mathrm{N}^{\circ} 11$ ). Los restantes grupos presentan tasas de crecimiento del empleo en el entorno del 20\%, con la excepción de los menos calificados que crecen por debajo del 10\%.

Para examinar si hay impactos potenciales del patrón de crecimiento de los ocupados en la distribución de ingresos, se procedió a estimar el cambio en el número de asalariados por hogar a nivel de centiles del ingreso per cápita. Los resultados se presentan en el Gráfico $\mathrm{N}^{\circ} 4$ y muestran que no hay efectos distributivos claros que se originen en los cambios en el empleo. Ello, puesto que el aumento de los ocupados por hogar se distribuye en forma pareja a lo largo de la distribución, sin presentar tendencias sistemáticas que pudieran inducir cambios en la desigualdad de los ingresos. 
De tal modo, el rol de los ingresos salariales en la caída de la desigualdad se originaría en los cambios en la estructura de los salarios antes que en el empleo asalariado.

CUADRO No $\mathrm{N}^{\circ}$ 11: ÍNDICE DE EMPLEO ASALARIADOS

\begin{tabular}{lrrrrrr}
\hline & \multicolumn{7}{c}{ Años de escolaridad } \\
\cline { 2 - 7 } & \multicolumn{1}{c}{$0-8$} & $9-11$ & 12 & $13-16$ & 17 y más & Todos \\
\hline 2000 & 100,0 & 100,0 & 100,0 & 100,0 & 100,0 & 100,0 \\
2003 & 93,3 & 104,0 & 110,5 & 106,4 & 110,6 & 104,0 \\
2006 & 108,6 & 119,7 & 141,1 & 118,3 & 118,0 & 121,9 \\
\hline
\end{tabular}

Nota: Incluye ocupados entre 25-65 que trabajan 30 o más horas semanales.

Fuente: Cálculos sobre la base de las encuestas Casen, años respectivos.

GRÁFICO Nº 4: $\quad$ CAMBIO EN EL NÚMERO DE ASALARIADOS POR HOGAR 2000 A 2006

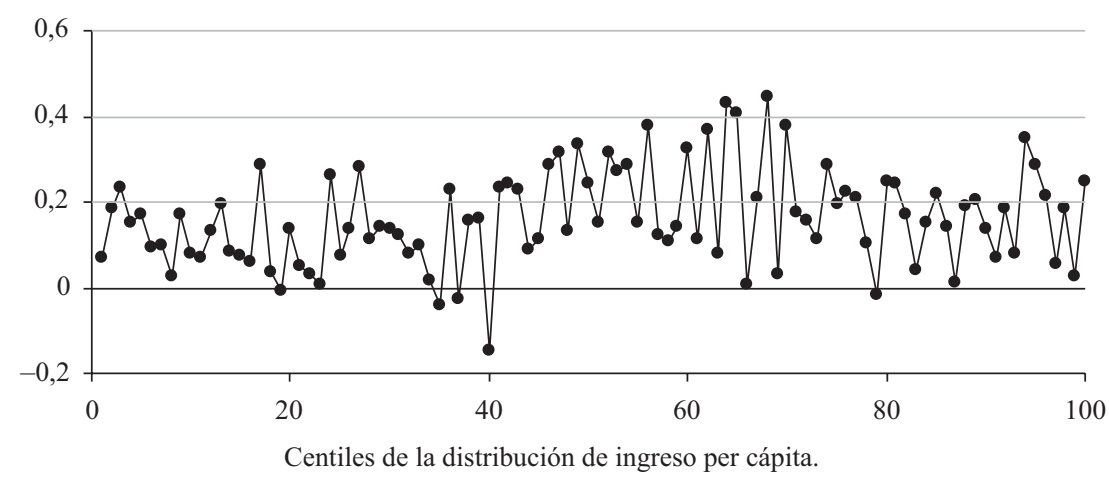

\section{El rol de la demografía}

La unidad relevante para estudiar la distribución de los ingresos es el hogar, puesto que sus miembros operan con una lógica de conjunto a efectos de la generación de los ingresos y de su posterior distribución al interior del hogar. Se sigue que aspectos demográficos, como es el tamaño del hogar y la composición de edades de sus miembros, pueden ser importantes en la explicación de los cambios distributivos. Ello es particularmente 
CUADRO No 12: DEMOGRAFÍA DE LOS HOGARES 1990-2006

\begin{tabular}{cccccccc}
\hline & $\begin{array}{c}\text { Número de } \\
\text { hogares, } \\
\text { índice }\end{array}$ & $\begin{array}{c}\text { Personas } \\
\text { por hogar }\end{array}$ & $\begin{array}{c}\text { 0-14 años } \\
\text { por hogar }\end{array}$ & $\begin{array}{c}\text { 65 y más años } \\
\text { por hogar }\end{array}$ & uniparentales & $\begin{array}{c}\text { \% hogares } \\
\text { unipersonales }\end{array}$ & $\begin{array}{c}\text { Número de } \\
\text { núcleos } \\
\text { por hogar }\end{array}$ \\
1990 & 100,0 & 3,99 & 1,14 & 0,26 & 21,7 & 6,9 & 1,22 \\
1996 & 113,1 & 3,89 & 1,09 & 0,29 & 20,3 & 7,0 & 1,17 \\
2000 & 122,0 & 3,82 & 1,04 & 0,30 & 22,1 & 7,9 & 1,19 \\
2003 & 129,7 & 3,73 & 0,95 & 0,30 & 23,7 & 8,7 & 1,20 \\
2006 & 136,7 & 3,70 & 0,86 & 0,34 & 26,0 & 8,7 & 1,22 \\
\hline
\end{tabular}

Nota: Todas las variables representan promedios, que incluyen a todos los hogares.

Fuente: Encuestas Casen, años respectivos.

cierto en el contexto de un país que exhibe fuertes cambios en la tasa de fecundidad, la esperanza de vida y la estructura de edades de la población. Estos desarrollos tienen un correlato a nivel de los hogares, con impactos directos e indirectos en la distribución de los ingresos.

Los principales rasgos de la demografía de los hogares en el período 1990-2006 se presentan en el Cuadro $\mathrm{N}^{\circ} 12$. En primer término, la tasa de crecimiento de los hogares excede largamente la tasa de crecimiento de la población (36,7\% vs 24,9\%), con una consiguiente caída en el tamaño medio de los hogares. Segundo, el promedio de menores de 15 años por hogar se reduce a un $75 \%$ de su nivel inicial, como reflejo de la rápida caída en la tasa de fecundidad que ha experimentado el país en las décadas recientes. Tercero, una mayor expectativa de vida está provocando aumentos en el número de adultos mayores por hogar.

También hay cambios en el tipo de hogares. Así, disminuye el porcentaje de hogares nucleares a favor de los hogares con un solo jefe (que en un $80 \%$ corresponde a jefes mujeres), así como de hogares unipersonales. Por otra parte, el número de núcleos familiares por hogar se mantiene estable en el período, hecho algo sorprendente si se considera que los incrementos en los ingresos y en el stock de vivienda podrían haber inducido procesos de autonomía residencial por parte de segundos núcleos familiares.

Demografía de los hogares y distribución de los ingresos

La relación entre la demografía de los hogares y la distribución de los ingresos se examina a nivel de deciles de ingreso en el Cuadro $\mathrm{N}^{\circ} 13$. Así, el tamaño del hogar está estrechamente relacionado con el decil de ingresos; los hogares en el decil más pobre son numerosos, alcanzando a 


\begin{tabular}{|c|c|c|c|c|c|c|}
\hline Deciles & $\begin{array}{l}\text { Personas } \\
\text { por hogar } \\
\quad(1)\end{array}$ & $\begin{array}{c}\text { 0-14 años } \\
\text { por hogar } \\
\quad(2)\end{array}$ & $\begin{array}{c}\text { 15-64 años } \\
\text { por hogar } \\
\text { (3) }\end{array}$ & $\begin{array}{c}65 \text { y más } \\
\text { por hogar } \\
(4)\end{array}$ & $\begin{array}{c}\text { Perceptores } \\
\text { (5) }\end{array}$ & $\begin{array}{c}\text { Inactivos/ } \\
\text { perceptores } \\
100 *(1-5) / 5\end{array}$ \\
\hline 1 & 4,51 & 1,65 & 2,65 & 0,21 & 1,00 & 3,47 \\
\hline 2 & 4,36 & 1,39 & 2,67 & 0,30 & 1,39 & 2,69 \\
\hline 3 & 4,19 & 1,16 & 2,65 & 0,38 & 1,58 & 2,25 \\
\hline 4 & 4,00 & 1,02 & 2,59 & 0,40 & 1,78 & 1,78 \\
\hline 5 & 3,81 & 0,84 & 2,57 & 0,41 & 1,85 & 1,53 \\
\hline 6 & 3,68 & 0,75 & 2,51 & 0,42 & 1,94 & 1,33 \\
\hline 7 & 3,50 & 0,67 & 2,43 & 0,39 & 1,92 & 1,13 \\
\hline 8 & 3,38 & 0,61 & 2,43 & 0,35 & 1,91 & 0,94 \\
\hline 9 & 3,22 & 0,54 & 2,38 & 0,30 & 1,89 & 0,86 \\
\hline 10 & 2,93 & 0,45 & 2,22 & 0,26 & 1,77 & 0,80 \\
\hline Total & 3,70 & 0,86 & 2,49 & 0,34 & 1,73 & 1,53 \\
\hline
\end{tabular}

Nota: Todas las variables representan promedios, que incluyen a todos los hogares.

Fuente: Encuestas Casen 2006.

4,5 miembros en promedio, mientras que aquellos en el decil más rico son relativamente pequeños, abarcando a menos de tres personas en promedio. La relación entre decil de ingresos y tamaño del hogar es continua, puesto que a medida que aumenta el primero, el segundo cae sistemáticamente. Obviamente se trata de una tendencia que opera a nivel de promedios, puesto que al interior de cada decil hay hogares de diferente tamaño.

Las diferencias de tamaño del hogar se explican básicamente por diferencias en el número de menores de 15 años. Un 75\% de la brecha en el tamaño promedio del hogar entre los deciles más rico y más pobre es explicado por diferencias en el número de menores. En cambio, el promedio de personas en edad de trabajar (15-65 años) así como de adultos mayores se mantiene sorprendentemente estable a nivel de los diferentes deciles de ingreso.

El porcentaje de hogares unipersonales crece con el decil de ingresos. Sólo un 3,5\% de los hogares del primer decil califica como unipersonales, proporción que sube a un 19,4\% en el decil décimo (Cuadro $\mathrm{N}^{\circ} \mathrm{A}-1$ en Anexo). La relación decreciente entre tamaño del hogar y decil de ingresos es explicada sólo parcialmente por los hogares unipersonales. La brecha de tamaño del hogar entre el decil más rico y el decil más pobre se reduce de 1,57 a 1,30 personas si se excluyen los hogares unipersonales ${ }^{20}$.

${ }^{20}$ En este último caso hay 4,64 personas como promedio en el hogar del decil pobre y 3,34 en el decil rico. 
Hay una evidente relación circular entre la variable demográfica y el ordenamiento de los hogares según deciles de ingreso per cápita.

Por una parte, el nivel de ingresos es un determinante de la variable demográfica. Aumentos de ingresos tienden a asociarse con una reducción en la tasa de fecundidad y con aumentos en la expectativa de vida, tal como lo muestra la evidencia de series de tiempo y aquella que compara estratos socioeconómicos en un determinado período.

Por otra parte, la variable demográfica es un determinante del ingreso per cápita. Ello opera a nivel de las decisiones familiares que inciden en la generación de los ingresos del hogar. Adicionalmente, pero no menos importante, el tamaño del hogar es directamente el denominador de la variable ingreso per cápita, introduciendo un nexo directo entre la demografía y la distribución de los ingresos.

Una variable síntesis de los factores demográficos es la tasa de dependencia, que es el cuociente entre el número de personas potencialmente inactivas desde una perspectiva laboral (menores de 15 y mayores de 65) y el número de personas potencialmente activas (15-65 años). Mientras en el decil más pobre hay 86,5 inactivos por cada 100 activos, en el decil más rico hay solo 34,2 inactivos por cada 100 activos; ésta es una diferencia de 2,55 veces en la tasa de dependencia. La relación entre inactivos y activos decrece en forma continua a nivel de los deciles de ingreso (Cuadro $\mathrm{N}^{\circ} \mathrm{A}-1$ en Anexo).

Una formulación alternativa del concepto de dependencia es la relación entre el número de personas que no reciben ingresos respecto de aquellas que sí lo hacen. Esta variante es más relevante cuando se analiza la distribución de los ingresos y se presenta en la última columna del Cuadro $\mathrm{N}^{\circ}$ 12. Por perceptor de ingreso se considera a toda persona que recibe algún ingreso monetario por encima del $20 \%$ del salario mínimo en el año respectivo. El corte es arbitrario y tiene por propósito excluir de la categoría de perceptores a quienes obtienen niveles de ingresos muy bajos (trabajos esporádicos, asignaciones familiares, etc.).

El valor promedio de la variable alcanza a 1,53 no perceptores de ingreso por cada perceptor de ingresos. Nuevamente, hay una relación inversa y continua entre la medida de dependencia y el decil de ingresos; en el decil rico hay 0,80 no perceptores por cada perceptor, mientras que en el decil más pobre dicha relación asciende a 3,47 veces.

\section{Efectos distributivos}

En el Cuadro $\mathrm{N}^{\circ} 14$ se presenta el cambio que experimentan las variables demográficas de los hogares en el período 2000 a 2006, a nivel de los 
deciles de ingreso. Los resultados establecen que hay cambios importantes en la estructura demográfica de los hogares y que estos cambios no son homogéneos a lo largo de la distribución de ingresos. Por tanto, hay bases para postular que la variable demográfica está jugando un rol en la dinámica distributiva.

Así, hay cambios importantes en el tamaño del hogar. Los hogares en la mitad inferior de la distribución presentan una caída promedio de 0,35 personas por hogar, equivalente a un 7\% de su nivel inicial en el año 2000. Por sí solo este efecto explicaría alrededor de una tercera parte del incremento del ingreso per cápita de los hogares en esta parte de la distribución, si todo lo demás se hubiese mantenido constante. En cambio, el tamaño promedio del hogar de los tres deciles superiores aumenta marginalmente, lo cual reduce su ingreso per cápita si los demás factores permanecieran constantes.

Las tendencias señaladas a nivel de deciles son bastante sólidas, tal como lo muestra la información a nivel desagregada. El Gráfico No 5 presenta el cambio en el tamaño de los hogares a nivel de centiles de la distribución de ingresos. Prácticamente todos los centiles hasta el percentil 60 presentan descensos en el tamaño del hogar, a la vez que la mayor parte de los centiles de la parte superior de la distribución muestran incrementos en la variable.

CUADRO No 14: CAMBIOS DEMOGRÁFICOS 2000-2006

\begin{tabular}{lcccccc}
\hline Deciles & $\begin{array}{c}\text { Personas } \\
\text { por hogar }\end{array}$ & $\begin{array}{c}0-14 \text { años } \\
\text { por hogar }\end{array}$ & $\begin{array}{c}15-64 \text { años } \\
\text { por hogar }\end{array}$ & $\begin{array}{c}65 \text { y más } \\
\text { por hogar }\end{array}$ & $\begin{array}{c}\text { Perceptores } \\
\text { Inactivos/ } \\
\text { perceptores } \\
(1-5) / 5\end{array}$ \\
\hline 1 & & & & 0,09 & $-0,46$ \\
2 & $-0,35$ & $-0,33$ & $-0,13$ & 0,11 & 0,09 & $-0,51$ \\
3 & $-0,33$ & $-0,37$ & $-0,10$ & 0,15 & 0,13 & $-0,58$ \\
4 & $-0,38$ & $-0,39$ & $-0,15$ & 0,17 & 0,18 & $-0,40$ \\
5 & $-0,35$ & $-0,29$ & $-0,20$ & 0,13 & 0,10 & $-0,40$ \\
6 & $-0,32$ & $-0,28$ & $-0,14$ & 0,10 & 0,14 & $-0,32$ \\
7 & $-0,21$ & $-0,20$ & $-0,14$ & 0,10 & 0,10 & $-0,12$ \\
8 & $-0,06$ & $-0,10$ & 0,02 & $-0,03$ & 0,12 & $-0,13$ \\
9 & 0,12 & $-0,06$ & 0,18 & $-0,04$ & 0,14 & $-0,17$ \\
10 & 0,14 & $-0,02$ & 0,16 & $-0,06$ & 0,14 & $-0,29$ \\
Total & 0,01 & $-0,12$ & 0,17 & $-0,06$ & 0,16 & \\
\hline
\end{tabular}

Nota: Todas las variables representan promedios, que incluyen a todos los hogares.

Fuente: Encuestas Casen, años respectivos. 
GRÁFICO N N 5: TASA CRECIMIENTO TAMAÑO DEL HOGAR 2000 A 2006

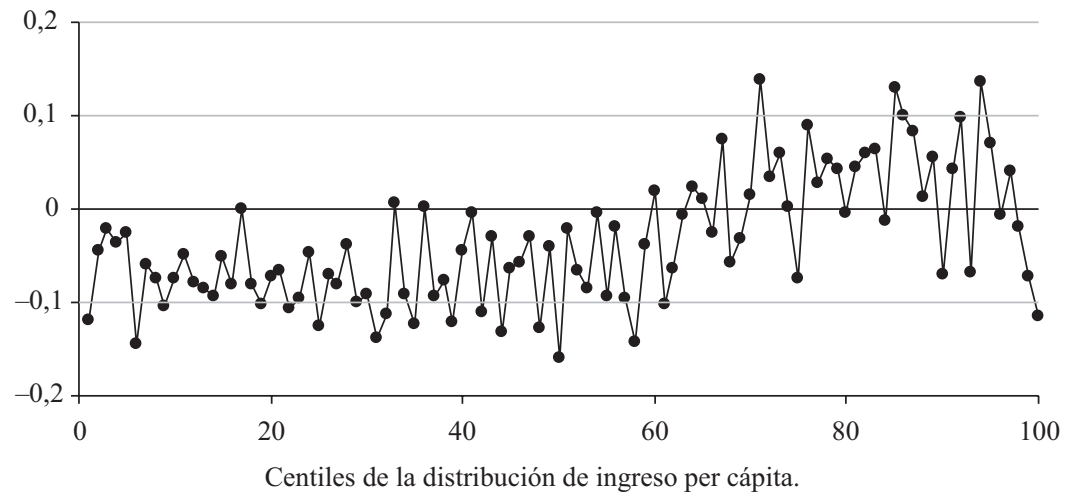

Los cambios en el tamaño de los hogares están muy correlacionados con los cambios en el número de menores por hogar. En particular, la reducción del tamaño del hogar en la mitad inferior de la distribución aparece casi enteramente explicada por una caída de similar magnitud en el número de niños por hogar. Este desarrollo puede vincularse a la evolución de la tasa de fecundidad por estrato socioeconómico.

En Larrañaga (2006) se muestra que las mujeres de estrato socioeconómico bajo lideraron la baja en la fecundidad en el período 1960-2000. En base a información de los censos de población se clasificó a las mujeres en cuartiles de nivel socioeconómico, definidos según nivel educacional. En el caso del cuartil primero, el número promedio de hijos por mujer desciende desde tasas cercanas a 6,0 a inicios de los sesenta a menos de 3,0 hacia inicios del 2000; mientras que el segundo cuartil muestra una caída de alrededor de 5 a 2,6 hijos por mujer en similar período de tiempo. Por su parte, las mujeres del estrato socioeconómico medio y alto también presentan caídas en la fertilidad, pero al interior de rangos más acotados: 4,0 a 2,2 y 3,0 a 1,9, respectivamente (Cuadro $\mathrm{N}^{\circ}$ A-2 en Anexo).

Una consecuencia de las tendencias señaladas es una relativa convergencia del comportamiento de la fertilidad según nivel socioeconómico. Mientras que a inicios de los sesenta la población de menor nivel socioeconómico tenía alrededor de 2,5 hijos más que la población de mayor nivel socioeconómico, a inicios de 2000 la brecha había disminuido a 1,0 hijo. 
No obstante lo anterior, podría parecer sorprendente la magnitud del cambio en el número de menores en el período más reciente, puesto que en el transcurso de seis años hay una caída del 17\% en el número de niños por hogar. Sin embargo, estos datos son consistentes con otras fuentes de información. Los datos de población del INE-Celade proyectan para el período 2000 a 2010 un descenso absoluto de $11 \%$ en el número de menores de 15 años de edad, sobre la base de información provista por los censos de población y las estadísticas vitales ${ }^{21}$. A tal respecto, considérese que alrededor de 10 de los 17 puntos de caída en el número de niños por hogar es explicada por una caída en el número absoluto de niños; mientras que los 7 puntos restantes se explican por el aumento en el número de hogares.

Por otra parte, a nivel de los deciles inferiores de ingresos se produce una caída en el número de personas en edad de trabajar por hogar, la cual tiende a compensarse con aumentos en el número de adultos mayores por hogar. En el caso de los deciles superiores ocurre la situación opuesta, puesto que aumenta la población en edad de trabajar y disminuyen los adultos mayores. Estos cambios podrían representar una tendencia desigualizante, puesto que alterarían la relación de dependencia en desmedro de los estratos inferiores de la distribución.

Sin embargo, la relación de dependencia entre perceptores y no perceptores informa que ello no es así, puesto que el cambio de la variable es relativamente pareja a nivel de los deciles (Cuadro $\mathrm{N}^{\circ} 14$, última columna). Ello ocurre en la medida que aumentos en la población de adultos mayores no representan necesariamente una mayor dependencia económica del hogar, dado que la mayor parte tiene acceso a pensiones contributivas o asistenciales.

De hecho, el porcentaje de perceptores de ingreso en relación con la población en cada tramo de edad es mayor en el segmento de mayores de 65 que en el segmento 15-64, aun si se excluye a los estudiantes. Efectivamente, un $86 \%$ de los mayores de 65 años de edad percibe ingresos (por arriba del umbral establecido), comparado con un $71 \%$ de aquellos entre 15 y 65 años de edad, excluyendo a los estudiantes ${ }^{22}$. Tal resultado ocurre por la amplia cobertura que tienen las pensiones en grupos que no participan del mercado del trabajo, como es el caso de las dueñas de casa que acceden a pensiones de montepío y pensiones asistenciales.

\footnotetext{
${ }^{21}$ Datos disponible en www.ine.cl.

${ }^{22}$ Datos provenientes de la encuesta Casen 2006.
} 


\section{Conclusiones}

Las conclusiones del trabajo pueden ser resumidas en seis puntos principales.

Primero, en el período 2000 a 2006 se produce una importante caída de la desigualdad de ingresos, puesto que la brecha entre los deciles más rico y más pobre cae de 38 veces a 28,5 veces, a la vez que el coeficiente de Gini desciende casi cuatro puntos porcentuales. La reducción de la desigualdad es un hecho sólido, tanto a nivel de los indicadores paramétricos como de la comparación de las funciones de distribución a través del análisis de dominancia. A pesar de lo anterior, el país sigue teniendo elevados niveles de desigualdad. La baja experimentada en el coeficiente de Gini representa menos del 15\% de la brecha de desigualdad que existe respecto del promedio de los países desarrollados.

Segundo, la caída de la desigualdad no se origina en la medición del año 2006, sino que ya está presente en el período 2000 a 2003. Por regla general, los datos del período conjunto 2000 a 2006 son consistentes entre sí, siendo sugerentes de movimientos de tendencia antes que efectos de un año en particular. De esta manera, la caída en la desigualdad no puede ser atribuida a los cambios realizados en el procedimiento muestral de la encuesta Casen del año 2006, si bien puede haber excepciones de carácter puntual. En esta materia hay que realizar un examen más detallado del nulo crecimiento del ingreso del decil más rico entre 2003 y 2006, así como su relación con la caída de la participación en el ingreso de los empleadores.

Tercero, la principal fuerza detrás de la reducción de la desigualdad de ingresos es una caída en la desigualdad de los salarios. La descomposición del cambio en el coeficiente de Gini informa que un 50\% de la caída en la desigualdad del ingreso de los hogares se debe a que los salarios de los hogares en los deciles inferiores crecieron más rápidamente que los salarios en los deciles superiores. Ese desarrollo se asocia a una compresión de la distribución salarial, tanto a nivel de trabajadores con diferente nivel de calificación (escolaridad) como al interior de grupos de ocupados de similar calificación.

Cuarto, en el período 2000 a 2006 se produce un significativo descenso en el tamaño de los hogares de la parte baja de la distribución de ingresos, el cual explica alrededor de un tercio del crecimiento de su ingreso per cápita en el período. Éste es un segundo factor que contribuye a la reducción de la desigualdad y se relaciona con la caída en el número de menores de 15 años, situación que tiene su origen en el descenso en la tasa de fecundidad. Este último ha sido el principal desarrollo demográfico de las 
últimas décadas y ha tenido como protagonista principal a las mujeres de estratos socioeconómicos bajos.

Quinto, la reciente reducción de la desigualdad ha posibilitado una fuerte baja en la tasa de pobreza. Este hecho contrasta con la experiencia de la década de los 90, cuando la caída de la pobreza se asociaba a un efecto crecimiento que era parejo a lo largo de la distribución de ingresos. Como fuera establecido, la reducción de la desigualdad se origina en desarrollos que tienen lugar en el mercado laboral y en las variables demográficas. Es probable que parte de los cambios en las variables laborales y demográficas refleje el impacto que la política social tiene sobre la acumulación de capital humano, social y productivo. Por otra parte, las transferencias monetarias del Estado son demasiado pequeñas y no tienen mayor incidencia en las estadísticas de pobreza y desigualdad.

Sexto, la caída de la pobreza es importante y es un hecho estadísticamente robusto. Sin embargo, tanto el nivel de la pobreza como su tasa de descenso en el tiempo dependen críticamente de la línea de pobreza que se utilice. Considerando que hay dudas razonables respecto del verdadero valor de la línea de la pobreza, se precisa tener mayor cuidado en la interpretación de los resultados de pobreza en el país.

\section{ANEXO}

CUADRO No A-1

\begin{tabular}{lcccc}
\hline Deciles & $\begin{array}{c}\text { \% hogar } \\
\text { unipersonal } \\
2006\end{array}$ & $\begin{array}{c}\text { Tasa } \\
\text { dependencia } \\
2006\end{array}$ & $\begin{array}{c}\text { \% hogar } \\
\text { unipersonal } \\
2006-2000\end{array}$ & $\begin{array}{c}\text { Tasa } \\
\text { dependencia } \\
2006-2000\end{array}$ \\
\hline 1 & & & & \\
2 & 3,5 & 86,5 & 1,0 & $-2,5$ \\
3 & 3,7 & 74,8 & 2,2 & $-7,0$ \\
4 & 4,1 & 67,8 & 1,6 & $-6,4$ \\
5 & 5,2 & 62,8 & 2,5 & $-3,7$ \\
6 & 6,9 & 54,3 & 3,7 & $-6,8$ \\
7 & 10,0 & 50,8 & 4,4 & $-6,7$ \\
8 & 11,5 & 49,0 & 0,3 & $-5,2$ \\
9 & 11,8 & 43,6 & $-4,3$ & $-5,0$ \\
10 & 13,6 & 39,0 & $-3,4$ & $-9,0$ \\
& 17,4 & 34,2 & $-1,4$ & $-5,8$ \\
Total & 9,4 & 54,5 & & \\
& & & & \\
\end{tabular}

Fuente: Encuestas Casen, años respectivos. 
CUADRO N ${ }^{\circ}$ A-2: FERTILIDAD POR NIVEL SOCIOECONÓMICO, CHILE 1960-2002

(Número de hijos por mujer)

\begin{tabular}{lcccc}
\hline & \multicolumn{4}{c}{ Nivel socioeconómico (cuartil años escolaridad) } \\
\cline { 2 - 5 } Cohorte 35-39 en año: & I & II & III & IV \\
\hline & & & & \\
1960 & 5,72 & 4,83 & 3,94 & 3,01 \\
1965 & 5,82 & 4,94 & 4,28 & 3,24 \\
1970 & 5,72 & 4,82 & 3,68 & 3,14 \\
1975 & 5,08 & 4,32 & 3,43 & 2,81 \\
1980 & 4,48 & 3,85 & 3,03 & 2,53 \\
1985 & 3,85 & 3,29 & 2,66 & 2,26 \\
1990 & 3,54 & 3,18 & 2,60 & 2,20 \\
1995 & 3,24 & 2,82 & 2,38 & 2,15 \\
2000 & 2,88 & 2,61 & 2,24 & 1,91 \\
& & & & \\
\hline
\end{tabular}

Fuente: Larrañaga (2006) sobre la base de los Censos de Población 2002 y 1992.

GRÁFICO A-1: CURVA DE LORENZ 2006

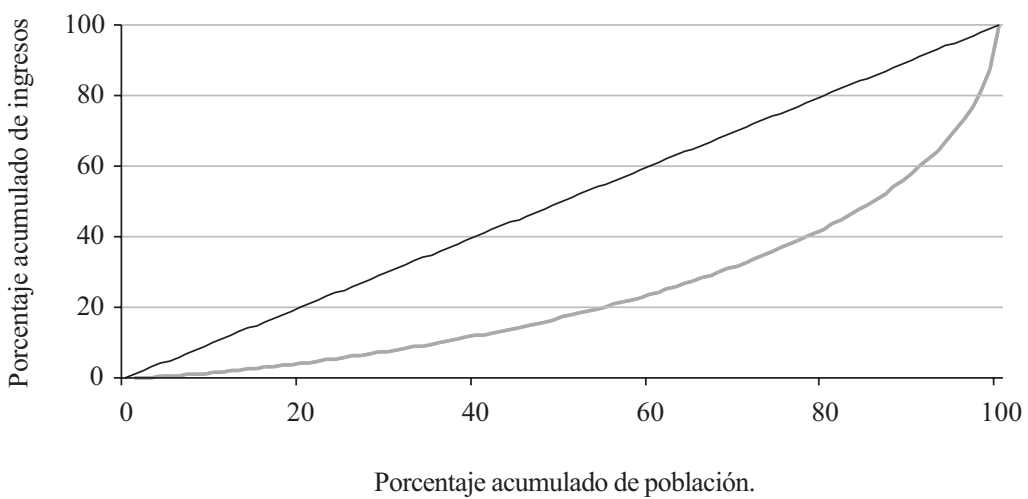


GRÁFICO A-2: $\quad$ FUNCIONES DISTRIBUCIÓN INGRESO P/C HOGAR 96; 00; 03 Y 06

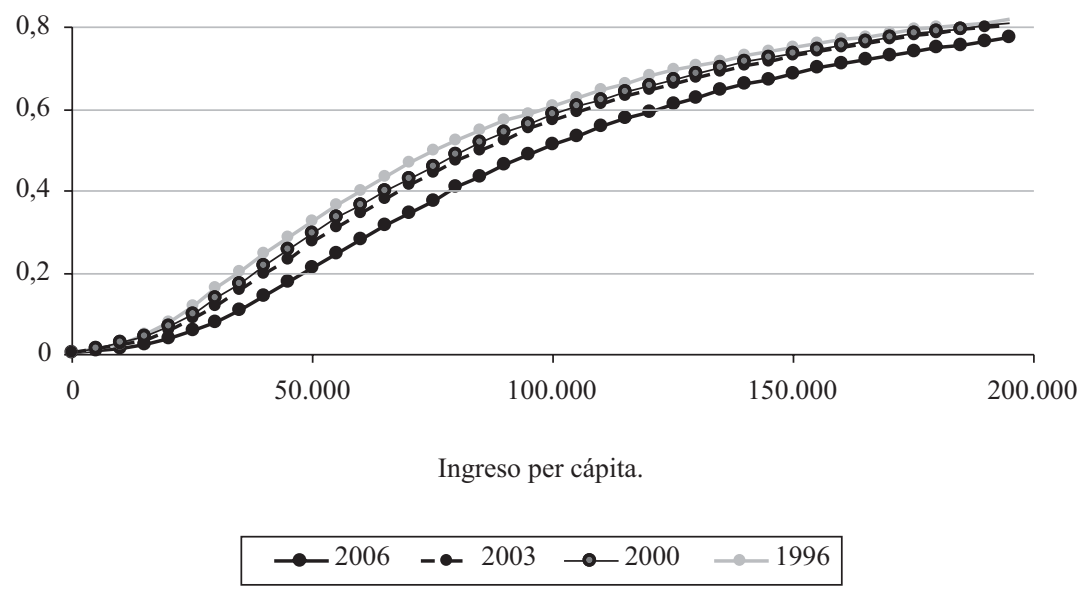

GRÁFICO A-3: $\quad$ DOMINANCIA ESTOCÁSTICA ORDEN 1 EN POBREZA; BASE 2006

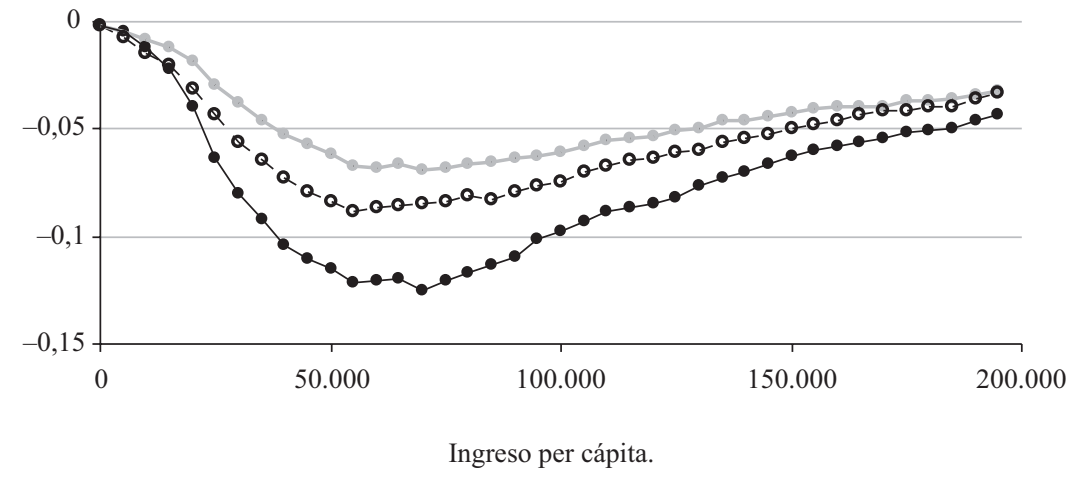


GRÁFICO Nº A-4: DOMINANCIA ESTOCÁSTICA ORDEN 2 EN POBREZA; BASE 2006

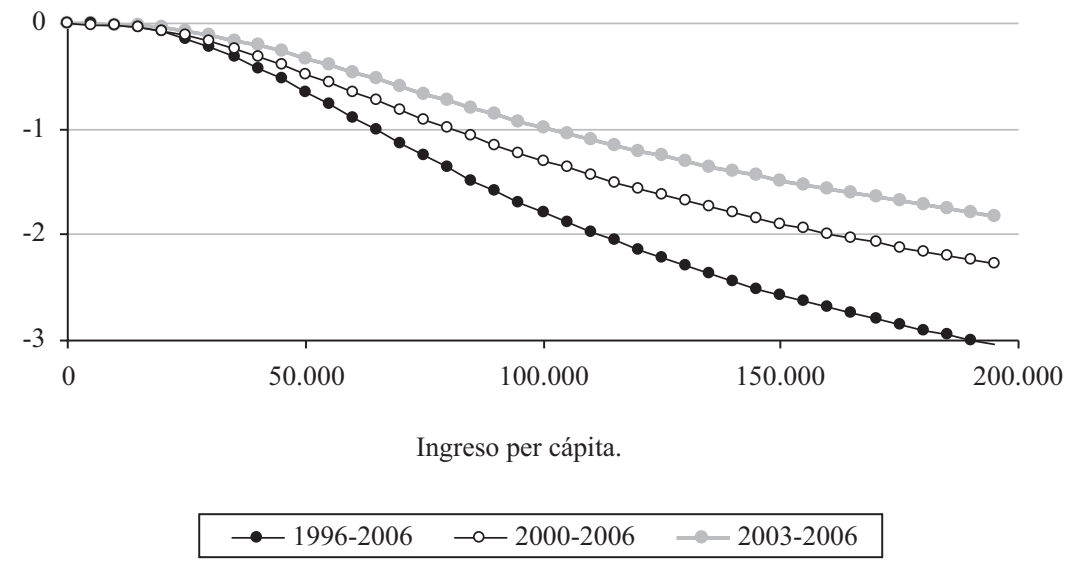

GRÁFICO No A-5: CRECIMIENTO REAL SALARIOS 2000 A 2006

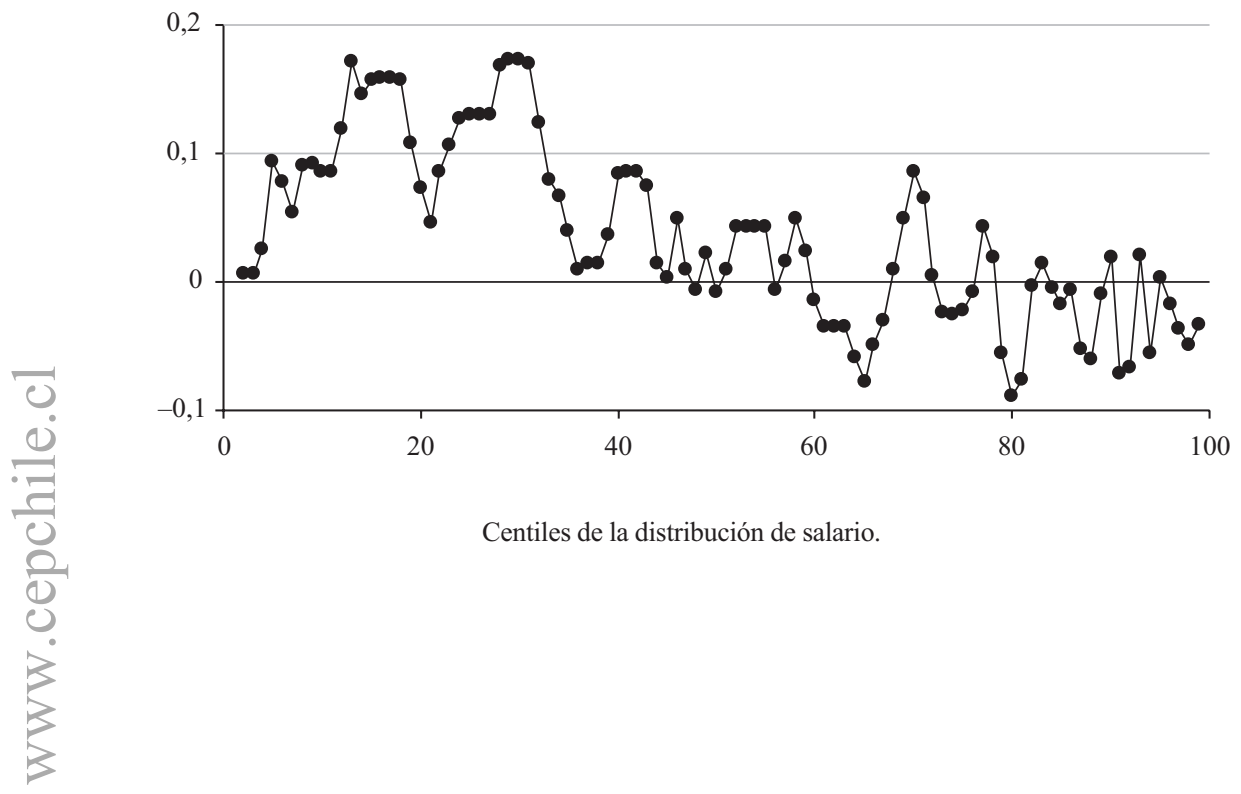




\section{REFERENCIAS BIBLIOGRÁFICAS}

Amiel, Y. y F. A. Cowell: Thinking about Inequality. Cambridge University Press, 1999.

Atkinson, A.: “On the Measurement of Inequality". En Journal of Economic Theory 2, 1970.

"On the Measurement of Poverty". En Econometrica, July 1987.

Cowell, F. A.: "Measurement of Inequality”. En A. B. Atkinson y F. Bourguignon (eds.), Handbook of Income Distribution. North Holland, 2000.

De Ferranti, D., G. Perry, F. Ferreira y M. Walton: Inequality in Latin America and the Caribbean: Breaking with History?. World Bank, 2003.

Datt, G. y M. Ravallion: "Growth and Redistribution Components of Changes in Poverty Measures”. Living Standards Measurement Papers 83, The World Bank, 1983.

Deaton, A.: The Analysis of Household Surveys. A Microeconometric Approach to Development Policy. John Hopkins, 1997.

Hausman, D. y M. McPherson: Economic Analysis and Moral Philosophy. Cambridge University Press, 1996.

Larrañaga, O.: "Pobreza, Crecimiento y Desigualdad: Chile 1987-92”. En Revista de Análisis Económico, noviembre de 1994.

“Comportamientos Reproductivos y Fertilidad, 1960-2003”. En J. Samuel Valenzuela, Eugenio Tironi y Timothy Scully (eds.): El Eslabón Perdido. Familia, Modernización y Bienestar en Chile. Santiago: Taurus, 2006.

“¿Qué Puede Esperarse de la Política Social en Chile?” Documento de Investigación, Departamento de Economía, Universidad de Chile, 2007a.

"La Medición de la Pobreza en Dimensiones Distintas del Ingreso". Serie de Estadísticas y Estudios Prospectivos 58, CEPAL, 2007b.

Mideplan: “Casen 2006, Documento Metodológico”. Santiago: Mideplan, 2007.

Rawls, J.: A Theory of Justice. Harvard University Press, 1971.

Sen, A.: On Economic Inequality. Edición expandida, con J. Foster. Oxford: Claredon Paperbacks, 1997.

Shorrocks, A.: “The Impact of Income Components on the Distribution of Family Incomes”. En The Quarterly Journal of Economics, Vol. 98(2), pp. 311-26, May 1983. 